\title{
Mesoscale Bounds in Viscoelasticity of Random Composites
}

\author{
Jun Zhang \\ Department of Mechanical Science \& Engineering \\ University of Illinois at Urbana-Champaign \\ Urbana, IL 61801, U.S.A. \\ e-mail: jzhng103@illinois.edu \\ Martin Ostoja-Starzewski \\ Department of Mechanical Science \& Engineering, \\ also Institute for Condensed Matter Theory and Beckman Institute \\ University of Illinois at Urbana-Champaign \\ Urbana, IL 61801, U.S.A. \\ e-mail: martinos@illinois.edu
}

April 8, 2015

\begin{abstract}
Under consideration is the problem of size and response of the Representative Volume Element (RVE) of spatially random linear viscoelastic materials. The model microstructure adopted here is the random checkerboard with one phase elastic and another viscoelastic, perfectly bonded everywhere. The method relies on the hierarchies of mesoscale bounds of relaxation moduli and creep compliances (Huet, 1995, 1999) obtained via solutions of two stochastic initial boundary value problems, respectively, under uniform kinematic and uniform stress boundary conditions. In general, the microscale viscoelasticity introduces larger discrepancy in the hierarchy of mesoscale bounds compared to elasticity, and this discrepancy grows as the time increases.
\end{abstract}

Keywords: random composite; viscoelasticity, representative volume element; mesoscale bounds

\section{Introduction}

The prediction of macroscopic properties of randomly structured heterogeneous materials is of major importance in many engineering applications. Evidently, any material displays heterogeneity on a micro scale and has properties depending on the scale of approximating continuum. The key issue, which commonly 
arises when dealing with structure-property relations of such materials is the validity of separation of scales of the continuum mechanical model

$$
\left.\begin{array}{l}
d \ll \\
d<
\end{array}\right\} L \ll L_{\text {macro }}
$$

Here $L$ is the size of the so-called Representative Volume Element (RVE), $d$ is the microscale, and $L_{\text {macro }}$ is the macroscale. The RVE is clearly set up in two basic cases (Ostoja-Starzewski, 2008): (i) a unit cell in a periodic microstructure, and (ii) a domain containing infinitely many microscale elements (e.g. inclusions) in a randomly structured medium.

This paper develops scale-dependent bounds on the effective response of random composites for a linear viscoelastic material with a two-phase, spatially random composition: a planar random checkerboard microstructure, in which one phase is viscoelastic and another elastic, with perfect bonding everywhere. We follow the approach that originated with (Huet, 1990; Sab, 1991, 1992): we deal with mesoscale (or apparent) material properties of finite size domains subjected to uniform boundary conditions of either kinematic or traction types. In general, these properties are random and mesoscale-dependent, and hence, such a domain is a Statistical Volume Element (SVE). As $L / d$ increases, the SVE tends towards the RVE - case (ii) above. Of interest is the evaluation of this trend from SVE to RVE in the time domain; the frequency domain properties of this model problem for a full range of volume fractions will be dealt with in a separate and more extensive paper. The SVE-to-RVE scaling issue has already been studied in many different settings besides linear elastic: conductivity, physically nonlinear elasticity, finite (thermo)elasticity, elasto-plasticity, permeability (Ostoja-Starzewski, 2008; Ostoja-Starzewski and Ranganathan, 2013). A theoretical basis for the viscoelastic setting was developed by Huet (1995, 1999) and this forms a stepping-stone for the present work, see also (Hazanov, 1996).

\section{Problem formulation}

\subsection{Random microstructure}

The random material is taken as a set of all the realizations $B(\omega)$ parametrized by sample events $\omega$ of the $\Omega$ space

$$
\mathcal{B}=\{B(\omega) ; \omega \in \Omega\} .
$$

Any realization $B(\omega)$ of the composite $\mathcal{B}=\{B(\omega) ; \omega \in \Omega\}$, while spatially disordered (i.e., heterogeneous), follows deterministic laws of mechanics.

The spatial (volume-type) averages will be denoted by the overbar, while the statistical (or ensemble) averages by $\langle\cdot\rangle$. That is, if we have a random $(n$ component, real valued) field $\Theta$ defined over some probability space $\{\Omega, \digamma, P\}$ (with $\digamma$ being a $\sigma$-field and $P$ a probability measure) and over some domain $V$ 
in $\mathbb{R}^{2}$ of volume $V$

$$
\Theta: \Omega \times X \rightarrow \mathbb{R}^{n},
$$

the said averages are

$$
\overline{\boldsymbol{\Theta}(\omega)} \equiv \frac{1}{V} \int_{v} \boldsymbol{\Theta}(\omega, \mathbf{x}) d V \quad\langle\boldsymbol{\Theta}(\mathbf{x})\rangle \equiv \int_{\Omega} \boldsymbol{\Theta}(\omega, \mathbf{x}) d P .
$$

As the random material we take a so-called random chessboard (or checkerboard) in two-dimensions, where each square cell of $M$ sites is occupied, independently of realizations at all other cells, with probability $p_{1}$ and $p_{2}$ by phases 1 and 2, respectively. Clearly, for a square lattice $L \times L=M$, the number of different realizations is $|\Omega|=2^{L \times L}$. Given the construction process, each $\omega$ occurs with probability $1 / 2^{L \times L}$. Technically speaking, it is a Bernoulli lattice process with the probability $p=\frac{1}{2}$. Fig. 1 shows sample realizations at three different scales and a nominal volume fraction $50 \%$. Indeed, the latter is the numerical setting in our computational mechanics reported below.

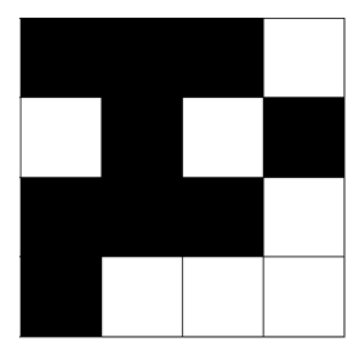

$L=4$

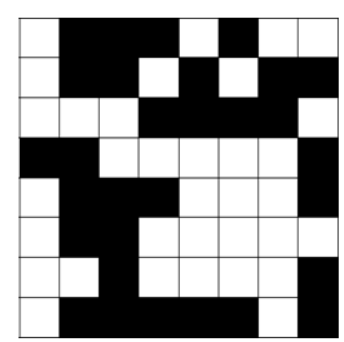

$L=8$

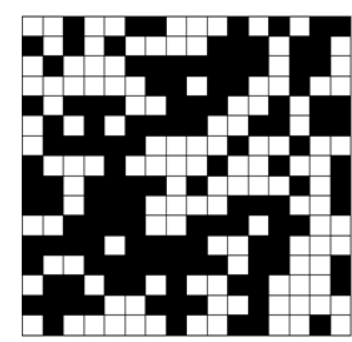

$L=16$

Figure 1: Sample realizations of the random, two-phase checkerboard on there $L \times L$ lattices.

In the following, it will be convenient to work with a mesoscale, a nondimensional parameter

$$
\delta=L / d \quad(d=\text { size of one site })
$$

in the range $[0, \infty)$, so that $\mathcal{B}_{L / d}$, a mesodomain, will be written $\mathcal{B}_{\delta}$, etc. Thus, $\delta=0$ signifies the pointwise description of the material, while $\delta \rightarrow \infty$ is the RVE limit. The mesoscale random material is a set of all the realizations $B_{\delta}(\omega)$ parametrized by sample events $\omega$ of the $\Omega$ space

$$
\mathcal{B}_{\delta}=\left\{B_{\delta}(\omega) ; \omega \in \Omega\right\} .
$$


Table 1: Properties of two phases

\begin{tabular}{|c|c|c|c|c|c|}
\hline & Type & $E$ & $\nu$ & $g_{1}$ & $\tau_{1}$ \\
\hline Mat A & Elastic & 60 & 0.3 & & \\
\hline Mat B & Viscoelastic & 30 & 0.3 & 0.9 & 0.25 \\
\hline
\end{tabular}

\subsection{Governing equations}

First, recall that the constitutive equations of linear viscoelastic solids in the time domain are expressed in terms of temporal Stieltjes convolutions:

$$
\begin{aligned}
& \sigma_{i j}(t)=\int_{0}^{t} r_{i j k l}(t-\tau): \epsilon_{k l}(\tau) d \tau, \\
& \epsilon_{i j}(t)=\int_{0}^{t} f_{i j k l}(t-\tau): \dot{\sigma_{k l}}(\tau) d \tau,
\end{aligned}
$$

where an overdot denotes the derivative with respect to time, while the tensors $r_{i j k l}(t)$ and $f_{i j k l}(t)$ are the relaxation modulus and creep compliance, respectively. With $\circ$ indicating the convolution integral, equations (7) and (8) can be expressed in a simplified indicial form:

$$
\begin{aligned}
& \sigma_{i j}=r_{i j k l} \circ \epsilon_{k l}, \\
& \epsilon_{i j}=f_{i j k l} \circ \sigma_{k l} .
\end{aligned}
$$

Linear viscoelasticity of our random material is implemented using the Prony series

$$
g_{R}(t)=1-\sum_{i=1}^{N} \overline{g_{i}}\left(1-e^{-\frac{t}{\tau_{i}}}\right),
$$

where $g_{R}(t)$ is the normalized shear modulus which starts from 1 at $t=0$ and then gradually decreases. $\overline{g_{i}}$ and $\tau_{i}$ are parameters that can be fitted to resemble the performance of a real relaxation behavior. For illustrative purposes in the present study, the linear viscoelasticity is assigned to one phase only and only one term in (11) is considered - this corresponds to a generalized Maxwell (i.e., Zener) model. The other phase is kept elastic. There is perfect bonding everywhere. Properties of both phases are given in Table 1. The relaxation moduli of both phases are also plotted in Fig. 2. From the figure, it can be concluded that the generated composite is like half rigid elastic solids mixed with half relatively "soft" viscoelastic gel, with the solids' modulus twice that of the gel.

The random heterogenous body $\mathcal{B}_{\delta}$ is described by the random fields of relaxation modulus tensor and creep compliance tensor

$$
\mathbf{r}: \Omega \times \mathbb{R}^{2} \rightarrow\left\{\mathbf{r}^{(1)}, \mathbf{r}^{(2)}\right\}
$$




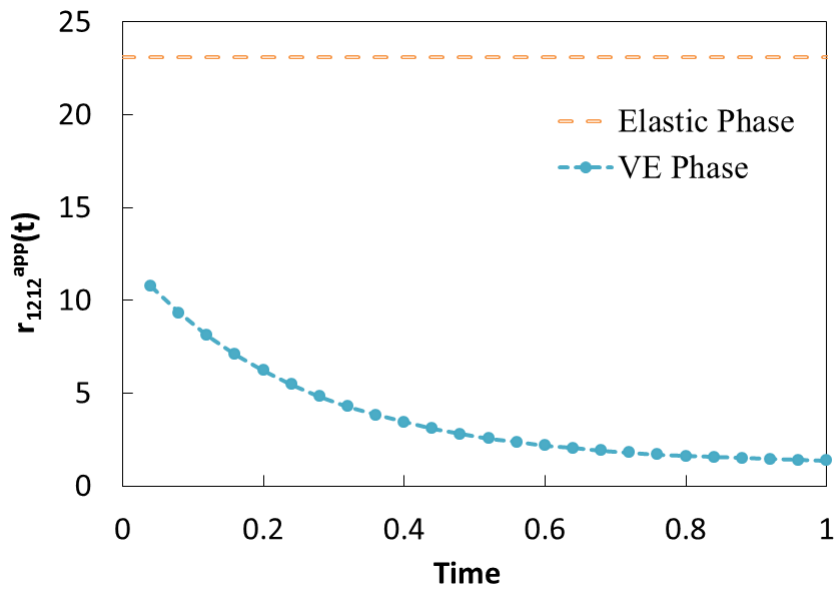

Figure 2: Relaxation moduli of both component phases.

or, in the ensemble sense,

$$
\left\{r_{i j k l}(\omega, \mathbf{x}, t) ; \omega \in \Omega, \mathbf{x} \in \mathbb{R}^{2}\right\} .
$$

Both phases (1 and 2) are assumed to be locally isotropic, and the random field to be white-noise. Then, the latter assumption results in a statistical isotropy of $\mathbf{r}$.

\section{Scale-dependent homogenization}

\subsection{The Hill-Mandel condition in viscoelasticity}

A proper definition of apparent properties should not only satisfy the original constitutive relation in an average sense, but also guarantee the equivalence of energetic with mechanical approaches in setting up constitutive equation. Taking the classical (Cauchy) continuum, rather than the micropolar (Cosserat) continuum, as the approximating model, this homogenization concept is expressed by the Hill-Mandel condition for viscoelastic materials

$$
\overline{\sigma_{i j}: \epsilon_{i j}}=\overline{\sigma_{i j}}: \overline{\epsilon_{i j}} .
$$

At finite scales, the equation (14) requires the body $\mathcal{B}_{\delta}$ to be loaded in a specific way on its boundaries, which can be accomplished by three different types of loadings:

- kinematic uniform boundary conditions (KUBC)

$$
u_{i}(x, t)=\epsilon_{i j}^{0}(t) x_{j}, \quad \forall x \in \partial B_{\delta},
$$


- static uniform boundary conditions (SUBC)

$$
t_{i}(x, t)=\sigma_{i j}^{0}(t) n_{j}, \quad \forall x \in \partial B_{\delta},
$$

- kinematic-traction (or mixed-orthogonal)

$$
\left(u_{i}(x, t)-\epsilon_{i j}^{0}(t) x_{j}\right)\left(t_{i}(x, t)-\sigma_{i j}^{0}(t) n_{j}\right)=0, \quad \forall x \in \partial B_{\delta},
$$

where $\sigma_{i j}^{0}(t)$ and $\dot{\epsilon}_{i j}^{0}(t)$ are the imposed constant tensors. [Actually, in viscoelasticity considered here, the loading (16) is quasi-static, but we call it "static" consistent with the works of Huet].

The above relies on the mean strain and stress theorems

$$
\begin{aligned}
& \overline{\epsilon_{i j}(t)}=\epsilon_{i j}^{0}(t), \\
& \overline{\sigma_{i j}(t)}=\sigma_{i j}^{0}(t),
\end{aligned}
$$

both of which hold for the mechanics problem at hand.

Given the random structure of $\mathcal{B}_{\delta}$ for any mesoscale $\delta,(15),(16)$, and (17) represent, respectively, three types of stochastic initial-boundary value problems.

\subsection{Hierarchies of mesoscale bounds in viscoelasticity}

In view of the mean strain and stress theorems, the mesoscale (apparent) relaxation modulus under uniform strain follows from (15)

$$
\overline{\sigma_{i j}}(\omega)=\overline{r_{i j k l}(\omega) \circ \epsilon_{k l}}=r_{i j k l, \delta}(\omega) \circ \epsilon_{k l}^{0},
$$

while the mesoscale (apparent) creep compliance under uniform stress follows from (16)

$$
\overline{\epsilon_{i j}}(\omega)=\overline{f_{i j k l}(\omega) \circ \sigma_{k l}}=f_{i j k l, \delta}(\omega) \circ \sigma_{k l}^{0} .
$$

In what follows, we do not use (17). Now, upon ensemble averaging, we obtain

$$
\text { from }(20):\left\langle\mathbf{r}_{\delta}(t)\right\rangle ; \quad \text { from }(21):\left\langle\mathbf{f}_{\delta}(t)\right\rangle \text {. }
$$

It has been shown by Huet $(1995,1999)$ that such relaxation moduli under the loading (15) and creep compliances under (16) of viscoelastic materials in the time domain satisfy, respectively, the following hierarchies on ensemble (statistical) averages:

$$
\begin{gathered}
\mathbf{r}^{e f f}(t) \leq \ldots \leq\left\langle\mathbf{r}_{\delta^{\prime}}(t)\right\rangle \leq\left\langle\mathbf{r}_{\delta}(t)\right\rangle \leq \ldots \leq\left\langle\mathbf{r}_{1}(t)\right\rangle, \quad \forall \delta^{\prime}>\delta, \quad \forall t>0, \\
\mathbf{f}^{e f f}(t) \leq \ldots \leq\left\langle\mathbf{f}_{\delta^{\prime}}(t)\right\rangle \leq\left\langle\mathbf{f}_{\delta}(t)\right\rangle \leq \ldots \leq\left\langle\mathbf{f}_{1}(t)\right\rangle, \quad \forall \delta^{\prime}>\delta, \quad \forall t>0 .
\end{gathered}
$$

Here $\mathbf{r}^{e f f}(t)$ and $\mathbf{f}^{e f f}(t)$ are the response tensors for an infinitely large domain (i.e., macroscale or the RVE), while $\left\langle\mathbf{r}_{1}(t)\right\rangle$ and $\left\langle\mathbf{f}_{1}(t)\right\rangle$ are the ensemble averaged 
response tensors for a mesoscale domain at $\delta=1$ (i.e., microscale). Effectively, the latter two are the Voigt and Reuss bounds in viscoelasticity.

The prescribed time dependence of strain $\epsilon_{k l}^{0}$ (or stress $\sigma_{k l}^{0}$ ) tensor in (15) [resp., (16)] is taken in the form of a Heaviside function $H(t)$, which then reduces the complicated convolution integral into a simple scalar product from which the apparent properties are inferred. As commonly done in viscoelasticity, the inertia effects under such nonstationary loadings are neglected. More specifically, we have:

- under KUBC (15), letting $\epsilon_{i j}^{0}(t)=\epsilon_{i j}^{0} H(t)$, yields

$$
\overline{\sigma_{i j}}=r_{i j k l}^{\delta}(\omega, t) \epsilon_{k l}^{0}
$$

- under $\operatorname{SUBC}(16)$, letting $\sigma_{i j}^{0}(t)=\sigma_{i j}^{0} H(t)$, yields

$$
\overline{\epsilon_{i j}}=f_{i j k l}^{\delta}(\omega, t) \sigma_{k l}^{0} \text {. }
$$

The computational study focuses on shear properties $r_{1212}^{\delta}$ and $f_{1212}^{\delta}$; they are simply related by $\sigma_{12}$ and $\epsilon_{12}$ without coupling any other stress and/or strain components. In the planar problem, the prescribed constant stress and strain tensors are set to be:

$$
\epsilon_{i j}^{0}=\epsilon_{12}^{0}\left[\begin{array}{cc}
0 & 1 \\
1 & 0
\end{array}\right] ; \quad \sigma_{i j}^{0}=\sigma_{12}^{0}\left[\begin{array}{ll}
0 & 1 \\
1 & 0
\end{array}\right]
$$

and

$$
r_{1212}^{\delta}(\omega, t)=\frac{\overline{\sigma_{12}(t)}}{\epsilon_{12}^{0}}, \quad f_{1212}^{\delta}(\omega, t)=\frac{\overline{\epsilon_{12}(t)}}{\sigma_{12}^{0}} .
$$

For the sake of general comparison, we also employ the analytic bounds that are obtained without any numerical simulation. Here, we apply the widely used Voigt and Reuss models, which correspond to the same iso-strain and iso-stress conditions as in elastic composites. However, the constitutive relations are now modified to viscoelasticity. For instance, in the Voigt model, we should have $\epsilon_{1}(t)=\epsilon_{2}(t)$, while $\sigma_{1}(t)=G_{1}(t) \circ \epsilon_{1}(t)$ and $\sigma_{2}(t)=G_{2}(t) \circ \epsilon_{2}(t)$ respectively. The subscripts 1 and 2 represent the two component phases.

Ideally, if the Voigt and Reuss models are taken under relaxation test, i.e.let $\epsilon_{1}(t)=\epsilon_{2}(t)=\epsilon_{0} H(t)$, and the creep test, i.e.let $\sigma_{1}(t)=\sigma_{2}(t)=\sigma_{0} H(t)$ respectively, the upper and lower bounds for both relaxation modulus and creep compliance should be developed respectively as well. However, the lower bound of the effective creep compliance in the Voigt model and lower bound of relaxation modulus in Reuss model cannot be solved explicitly due to the convoluted integral. The analytic solutions we can derive are:

- The upper bound on relaxation modulus, determined by the iso-strain condition of Voigt model (i.e., via arithmetic average of shear modulus of both phases)

$$
G_{A}(t)=\alpha G_{1}(t)+(1-\alpha) G_{2}(t) .
$$


- The upper bound on creep compliance, determined by the iso-stress condition of Reuss model (i.e., via arithmetic average of shear compliance of both phases)

$$
J_{A}(t)=\alpha J_{1}(t)+(1-\alpha) J_{2}(t) .
$$

These formulas are based on the quasi-elastic moduli $G_{i}(t)$ and $J_{i}(t)$ [calculated by running a simulation of creep test from $G_{i}(t)$ ] of the component phases $(i=1,2)$.

Since the relaxation modulus and creep compliance are reciprocals of each other, the lower bound of relaxation modulus is easily obtained by just inverting the upper bound of creep compliance. The same approach can be applied to creep compliance as well.

\subsection{Computational Results}

The time dependent behavior of viscoelastic planar random checkerboard are simulated through finite element methods using ABAQUS. Plane stress condition is assumed and each square gain (pixel) in the microstructure is discretized into 16 elements ( 4 by 4 ), this criteria is chosen when stable and converged average behaviors are attained. To better resolve the stress and strain distributions at interface of two phases, we use quadratic elements instead of bilinear elements. Figure 3 exhibits the example of mesh density study at $\delta=4$, in which the legend "Ix4" means the simulation is conducted at mesoscale 4 and "CPS8" stands for the 8-node plane stress quadratic elements. "MeshX" denotes the density at which every pixel is descretized in each direction( $\mathrm{x}$ and y). It is observed that, as the mesh density gets larger than 4 , there is almost negligible difference in the averaged response of the material.

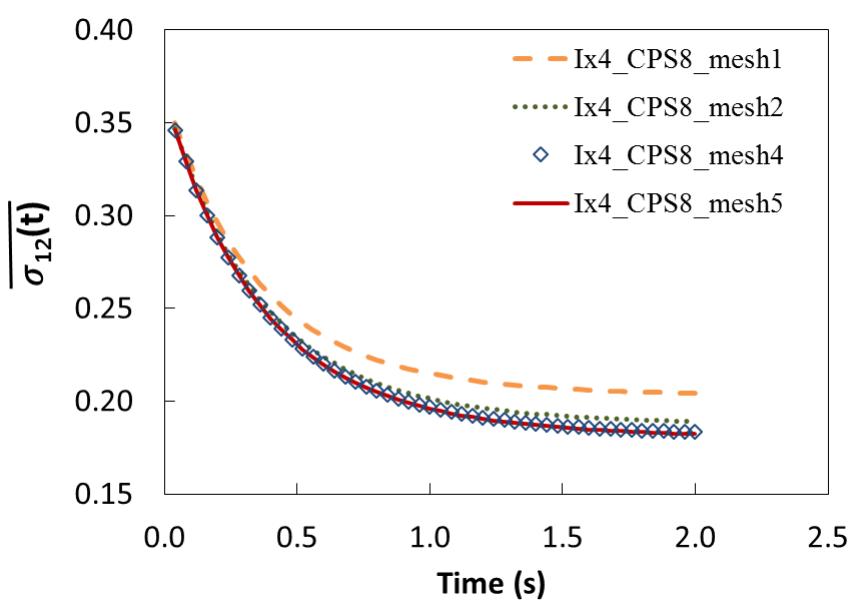

Figure 3: Mesh density study for realization at $\delta=4$. 
Stress and strain distributions under KUBC and SUBC are shown in Fig. 4-9 at all scales. Under the KUBC, displacements are applied on all boundary nodes suddenly at $t=0$ and then held constant. The entire structure is then experiencing stress relaxation for $t>0$ due to viscoelasticity. Stresses are distributed heterogeneously because of the discrepancy in materials properties between elastic and viscoelastic phases. As time increases, the viscoelastic phase softens and shows a steeper drop in stress as compared to the elastic one. At the same time, the modulus contrast between two phases also increases as one phase relaxes and this promotes high stress concentrations at the bonding interfaces.

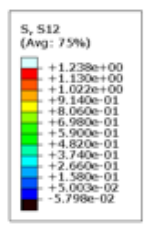

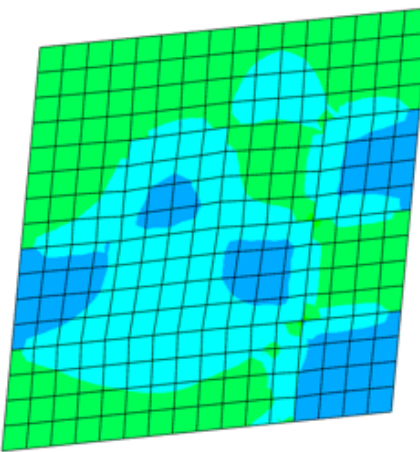

(a)

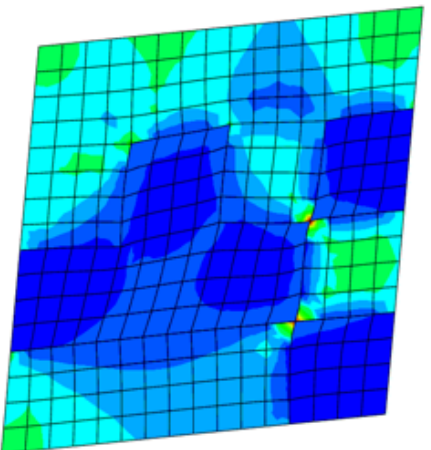

(b)

Figure 4: Stress distribution under KUBC of one SVE at mesoscale $\delta=4$. (a) $\mathrm{t}=0.04(\mathrm{~b}) \mathrm{t}=2.0$.

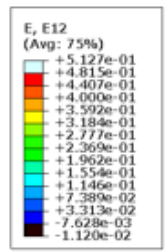

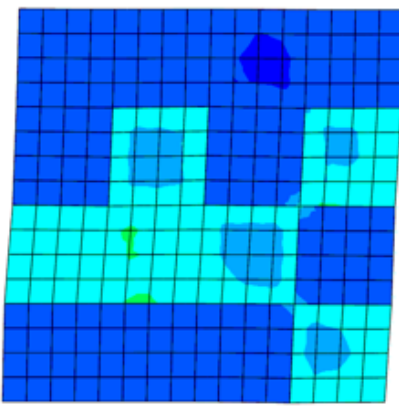

(a)

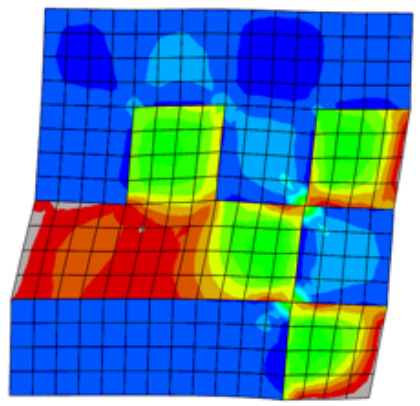

(b)

Figure 5: Strain distribution under SUBC of one SVE at mesoscale $\delta=4$. (a) $\mathrm{t}=0.04(\mathrm{~b}) \mathrm{t}=2.0$.

For the case of SUBC, the degrees of freedom of the lower right corner node in y-direction are restricted in order to eliminate rigid body rotation. Similar to 


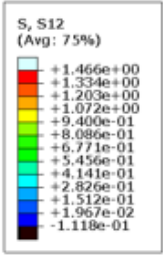

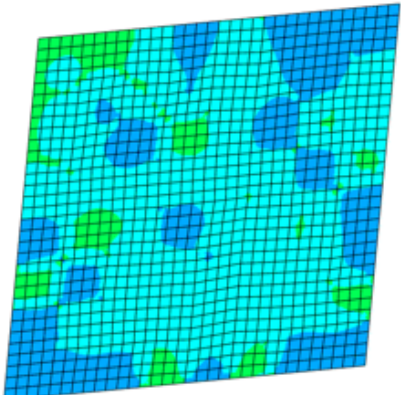

(a)

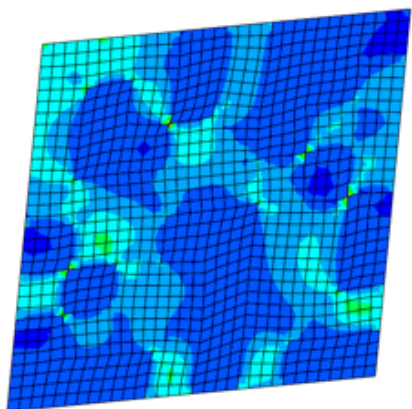

(b)

Figure 6: Stress distribution under KUBC of one SVE at mesoscale $\delta=8$. (a) $\mathrm{t}=0.04(\mathrm{~b}) \mathrm{t}=2.0$.

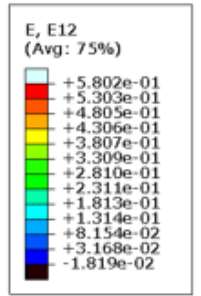

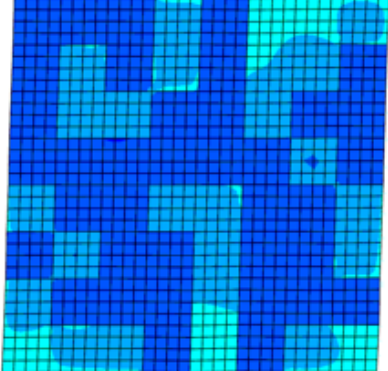

(a)

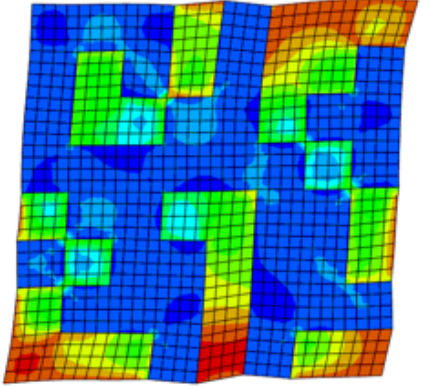

(b)

Figure 7: Strain distribution under SUBC of one SVE at mesoscale $\delta=8$. (a) $\mathrm{t}=0.04(\mathrm{~b}) \mathrm{t}=2.0$.

what is observed under KUBC, as the viscoelastic phase gets weaker, it creeps more and accumulates more strain. The elastic phase is fixed in modulus and the strain level in it does not change much, except for regions that are close to interfaces.

The mesoscale properties for each single realization are computed according to (25) and (26) and then,upon Monte Carlo sampling of the set of realizations of random checkerboards, are subjected to ensemble averaging, see Fig. 10. Note the consistent trend as the mesoscale parameter $\delta$ increases, which also matches the theoretical hierarchies of bounds as predicted in (23) and (24). As $\delta$ continues to increase and finally approaches the RVE, the obtained apparent properties converge to effective properties which do not vary with mesoscale and boundary conditions anymore.

Recall that in the elastic analysis, the size of RVE can be determined by observing the convergence of two mesoscale bounds as $\delta$ increases. This would still 


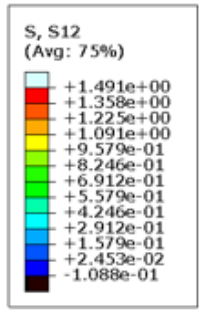

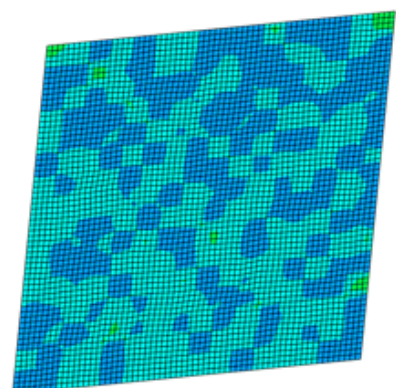

(a)

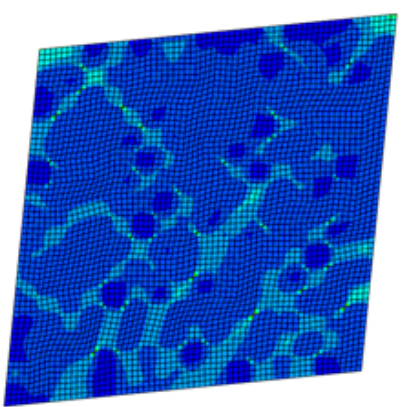

(b)

Figure 8: Stress distribution under KUBC of one SVE at mesoscale $\delta=16$. (a) $\mathrm{t}=0.04(\mathrm{~b}) \mathrm{t}=2.0$.

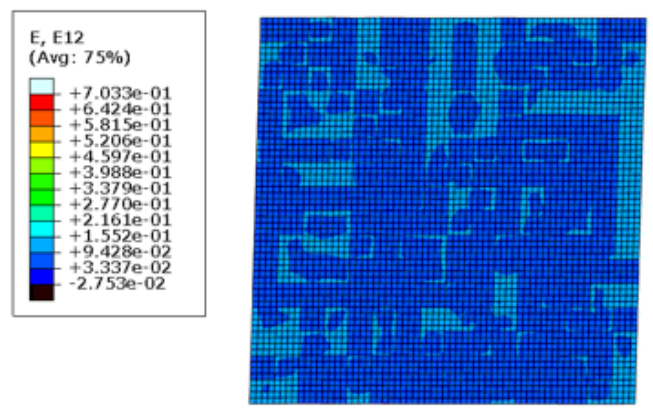

(a)

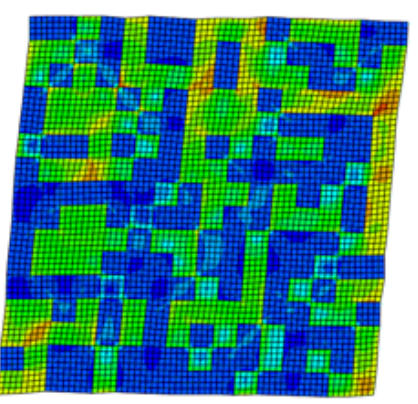

(b)

Figure 9: Strain distribution under SUBC of one SVE at mesoscale $\delta=16$. (a) $\mathrm{t}=0.04(\mathrm{~b}) \mathrm{t}=2.0$.

be favored for viscoelasticity. However, the difficulty is that the interrelation between relaxation modulus and compliance in the time domain for viscoelasticity does not satisfy the simple inversion, i.e. $f_{1212}(t) \neq r_{1212}(t)^{-1}$. Analytically, the relaxation modulus and the creep compliance are related through a convolution integral (Ferry, 1980)

$$
\int_{0}^{t} \mathbf{r}(t-\tau) \mathbf{f}(\tau) d \tau=t, \quad \forall t>0
$$

While a direct solution of Equation (30) is unwieldy and is not desirable, note that (Park and Kim, 1999) introduced an approximate formula relating the relaxation modulus with the creep compliance

$$
\mathbf{r}(t) \mathbf{f}(t)=\frac{\sin n \pi}{n \pi} .
$$



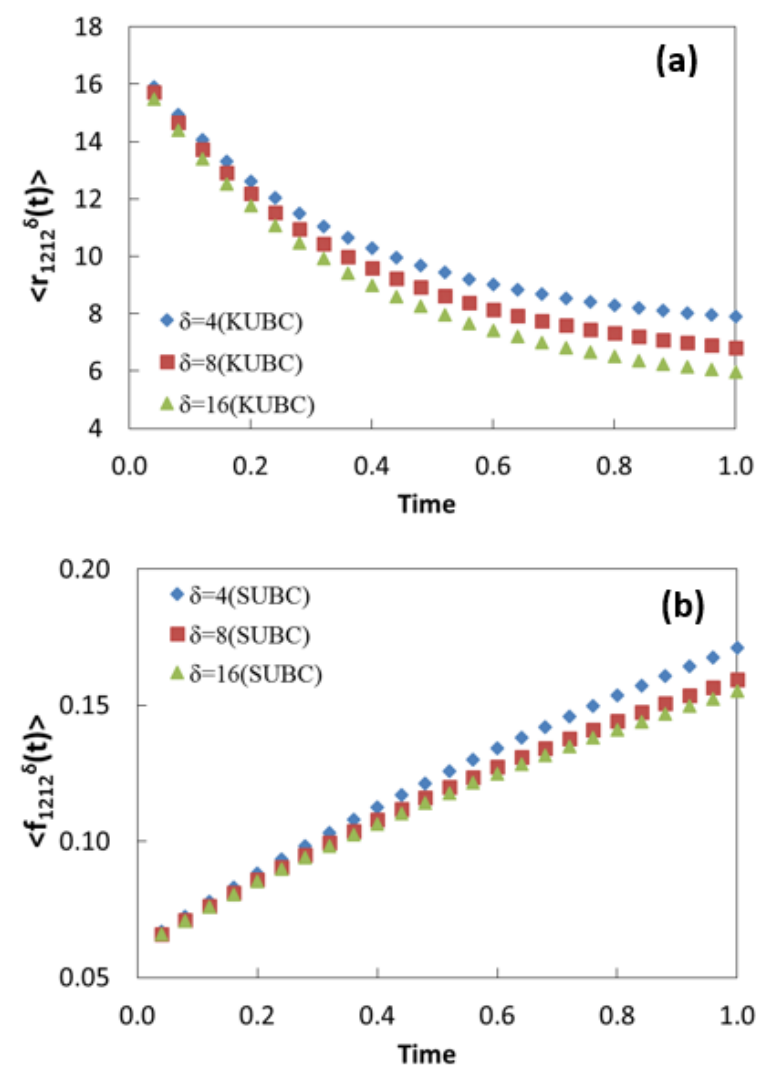

Figure 10: (a) Mesoscale (apparent) relaxation moduli and (b) Mesoscale (apparent) creep compliances.

Here $n=\left|\frac{d \log F(\tau)}{d \tau}\right|_{\tau=t}$ is the $\log -\log$ slope of source function $F(\tau)$, where $F(\tau)$ can be either $\mathbf{r}(t)$ or $\mathbf{f}(t)$. The approximation in (31) is considered to be good as long as the logarithm of source function is smooth and slowly varying in slope.

By using (31), the apparent relaxation moduli from KUBC and SUBC can now be plotted together in Fig. 11 The mesoscale properties from both boundary conditions are clearly bounded by bounds (28)-(29). The convergence of two mesoscale bounds in the time domain, for $\delta$ increasing, is now clearly observed. The actual effective properties of plane checkerboard with viscoelastic phase at nominal probability 0.5 at RVE fall between the narrow bounds provided by mesoscale properties evaluated at $\delta=16$.

Mesoscale bounds are also plotted at two time instants (0.2 and 1.0) in function of $\delta$ in Fig. 12. For comparison, the elastic case with the same microstruc- 


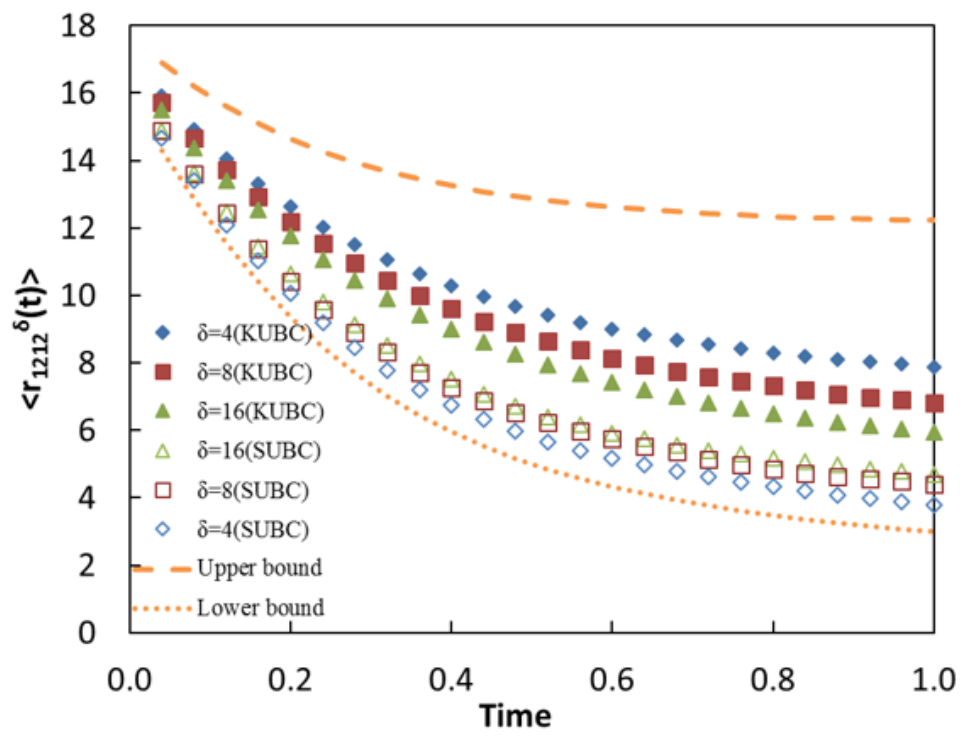

Figure 11: Mesoscale (apparent) relaxation moduli and converted apparent creep compliances in function of time. The bounds (28)-(29) are also shown.

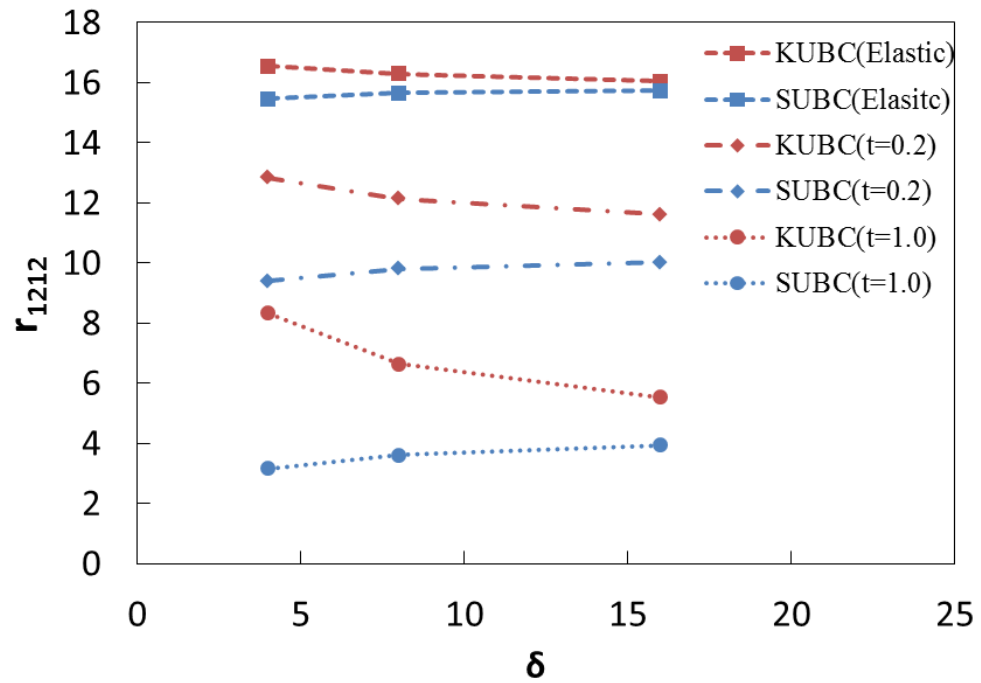

Figure 12: Mesoscale bounds in function of mesoscale for several time instances

ture is also included; these two top curves can be regarded as the apparent viscoelastic properties measured at $t=0$ (i.e., when the loading is just being 
applied). This is implemented by setting $g_{1}$ and $\tau_{1}$ in Table 1 to be 0 . Generally, viscoelasticity introduces larger discrepancy in the hierarchy of mesoscale bounds compared to elasticity, and this discrepancy grows as the time increases.

\section{Conclusion}

In this report, the methodology for obtaining scale-dependent properties of linear viscoelastic composites in time domain (Huet, 1995, 1999) has been pursued. Hierarchies of mesoscale bounds are determined numerically in the time domain for a planar random viscoelastic material whose microstructure is modeled as the random checkerboard where one phase is viscoelastic (Zener type) and another elastic. The bounds, defined in terms of relaxation and compliance, are obtained via solutions of two stochastic initial boundary value problems set up, respectively, for kinematic and traction (static) boundary conditions.The results are illustrated by finite element simulations of random checkerboards for several mesoscales. While both phases (1 and 2) of the microstructure are assumed to be locally isotropic, and the random field to be white-noise, thus assuring the statistical isotropy of the $\mathbf{r}$ field, the mesoscale response of any given realization (and hence in the ensemble sense) is generally not isotropic. This work provides the basis for analysis in the frequency domain and development of scaling laws, which will be reported in the next paper.

The method presented in this paper can be used to assess the scale dependent passage from SVE to RVE in (i) a wide range of viscoelastic composites with other microstructural morphologies (e.g., with spatial correlations), (ii) a Cosserat rather than a Cauchy continuum homogenization (Trovalusci et al., 2014), and (iii) in material systems with micro/nanoscale viscous phenomena where the second law of thermodynamics is violated (Ostoja-Starzewski and Malyarenko, 2014).

\section{Acknowledgment}

This material is based upon work supported by the NSF under grant 1362146.

\section{References}

Ferry, J.D. (1980), Viscoelastic Properties of Polymers (3rd ed.), Wiley, NY.

Huet, C. (1990), Application of variational concepts to size effects in elastic heterogeneous bodies. J. Mech. Phys. Solids 38(6), 813-841.

Huet, C. (1995), Bounds for the overall properties of viscoelastic heterogeneous and composite materials. Arch. Mech. 47(6), 1125-1155.

Huet, C. (1999), Coupled size and boundary-condition effects in viscoelastic heterogeneous and composite bodies. Mech. Mater. 31, 787-829.

Hazanov, S. (1996). Viscoelastic minimum principles revisited. J. Appl. Mech. 63, 551-554.

Ostoja-Starzewski, M. (2008), Microstructural Randomness and Scaling in Mechanics of Materials, Chapman \& Hall/CRC Press. 
Ostoja-Starzewski and A. Malyarenko, A. (2014), Continuum mechanics beyond the second law of thermodynamics. Proc. R. Soc. A 470, 20140531.

Ostoja-Starzewski, M. and Ranganathan, S.I. (2013), Scaling and Homogenization in Spatially Random Composites, Ch. 2 in Mathematical Methods and Models in Composites (Ed. V. Mantic), World Scientific, 61-102.

Park, S.W. and Kim, Y.R. (1980), Interconversion between relaxation modulus and creep compliance for viscoelastic solids. J. Mater. Civil Eng. 11, $76-82$.

Sab, K. (1991), Principe de Hill et homogénéisation des matériaux aléatoires. C. R. Acad. Sci. Paris II 312, 1-5.

Sab, K. (1992), On the homogenization and the simulation of random materials. Europ. J. Mech., A/Solids 11,c 585-607.

Trovalusci, P., Ostoja-Starzewski, M., de Bellis, M.L. and Muralli, A. (2014), Homogenization of random composites as micropolar continua. Europ. J. Mech./A: Solids 49, 396-407.

\section{Figures}

Fig. 1: Sample realizations of the random, two-phase checkerboard on three $L \times L$ lattices.

Fig. 2: Relaxation moduli of both component phases.

Fig. 3: Mesh density study for realization at $\delta=4$.

Fig. 4: Stress distribution under KUBC of one SVE at mesoscale $\delta=4$. (a) $\mathrm{t}=0.04(\mathrm{~b}) \mathrm{t}=2.0$

Fig. 5: Strain distribution under SUBC of one SVE at mesoscale $\delta=4$. (a) $\mathrm{t}=0.04(\mathrm{~b}) \mathrm{t}=2.0$

Fig. 6: Stress distribution under KUBC of one SVE at mesoscale $\delta=8$. (a) $\mathrm{t}=0.04(\mathrm{~b}) \mathrm{t}=2.0$

Fig. 7: Strain distribution under SUBC of one SVE at mesoscale $\delta=8$. (a) $\mathrm{t}=0.04(\mathrm{~b}) \mathrm{t}=2.0$

Fig. 8: Stress distribution under KUBC of one SVE at mesoscale $\delta=16$. (a) $\mathrm{t}=0.04(\mathrm{~b}) \mathrm{t}=2.0$

Fig. 9: Strain distribution under SUBC of one SVE at mesoscale $\delta=16$. (a) $\mathrm{t}=0.04(\mathrm{~b}) \mathrm{t}=2.0$

Fig. 10: (a) Mesoscale (apparent) relaxation moduli and (b) Mesoscale (apparent) creep compliances.

Fig. 11: Mesoscale (apparent) relaxation moduli and converted apparent creep compliances in function of time. The bounds (28)-(29) are also shown.

Fig. 12: Mesoscale bounds in function of mesoscale for several time instances. 


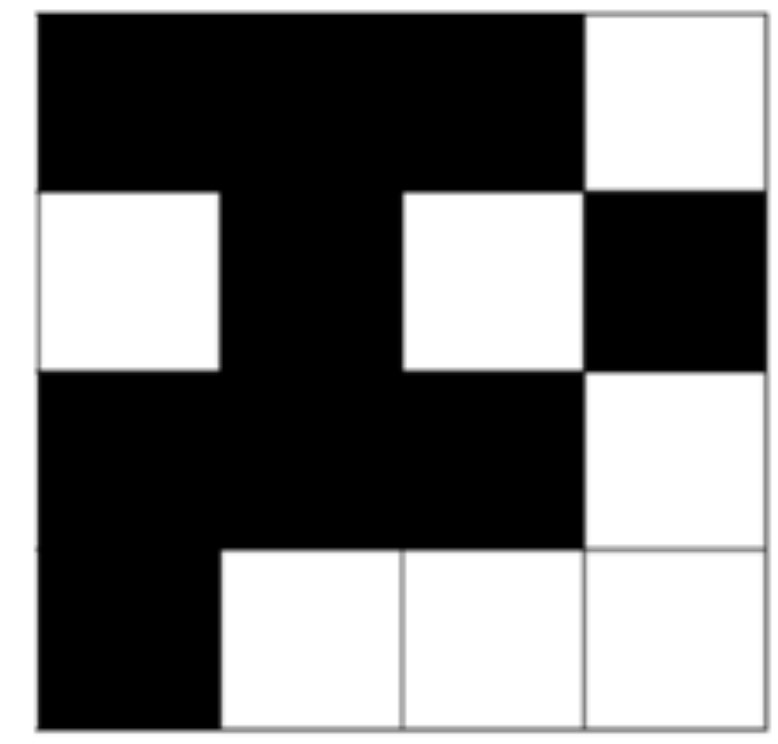

$L=4$

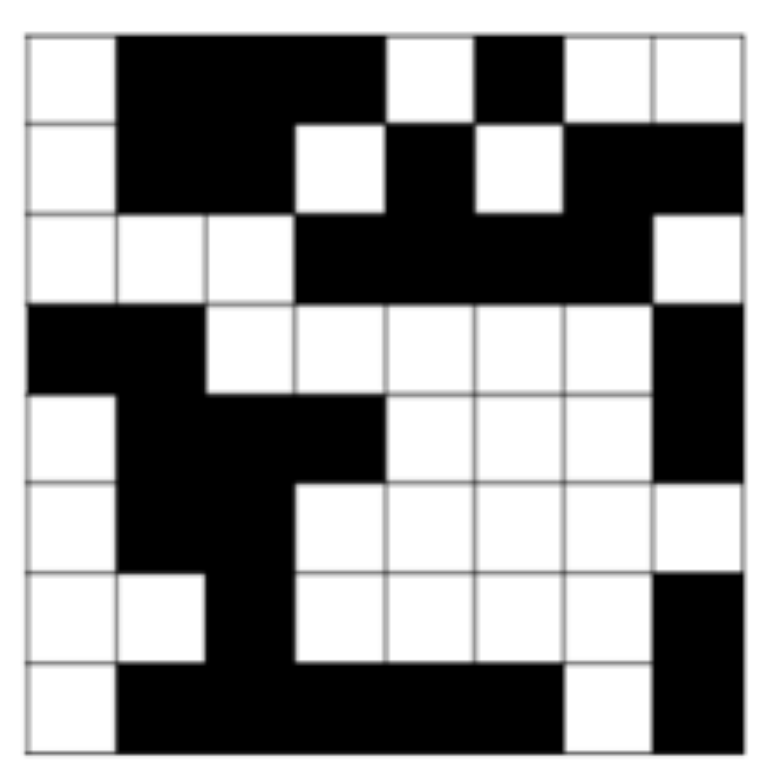

$$
L=8
$$

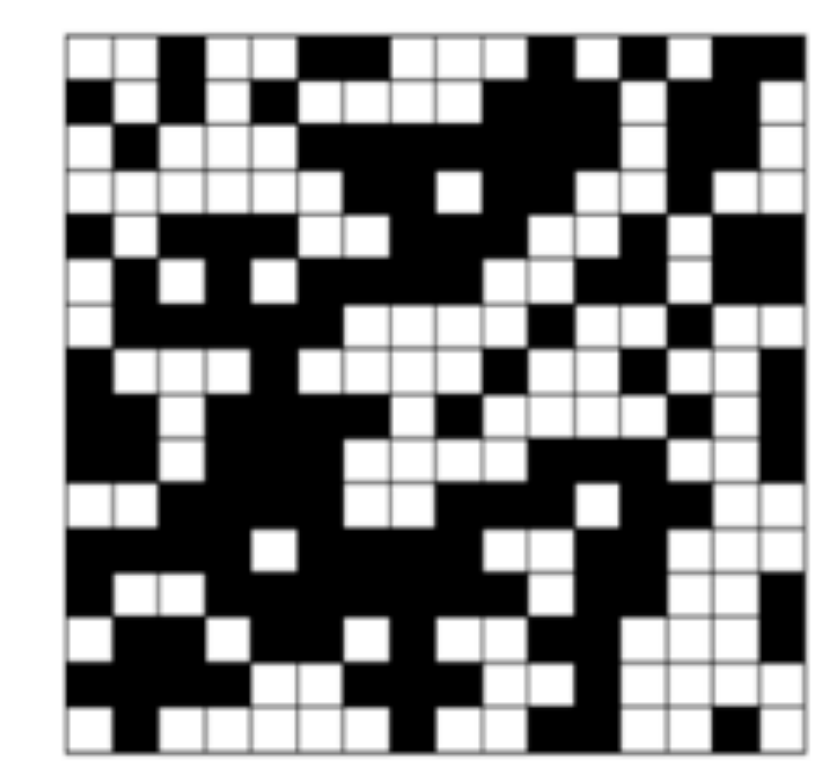

$$
L=16
$$

\section{Click here to download high resolution image}




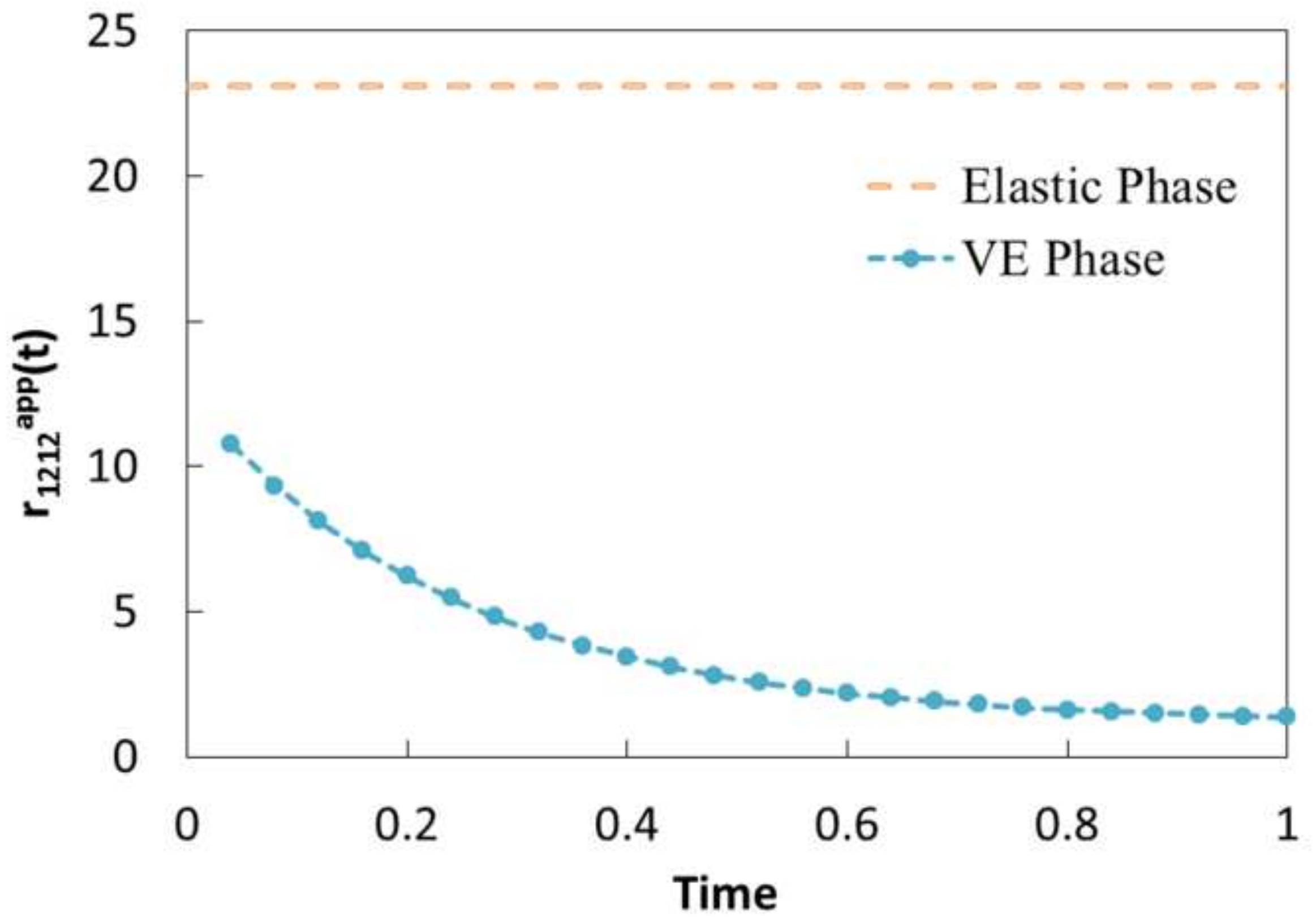




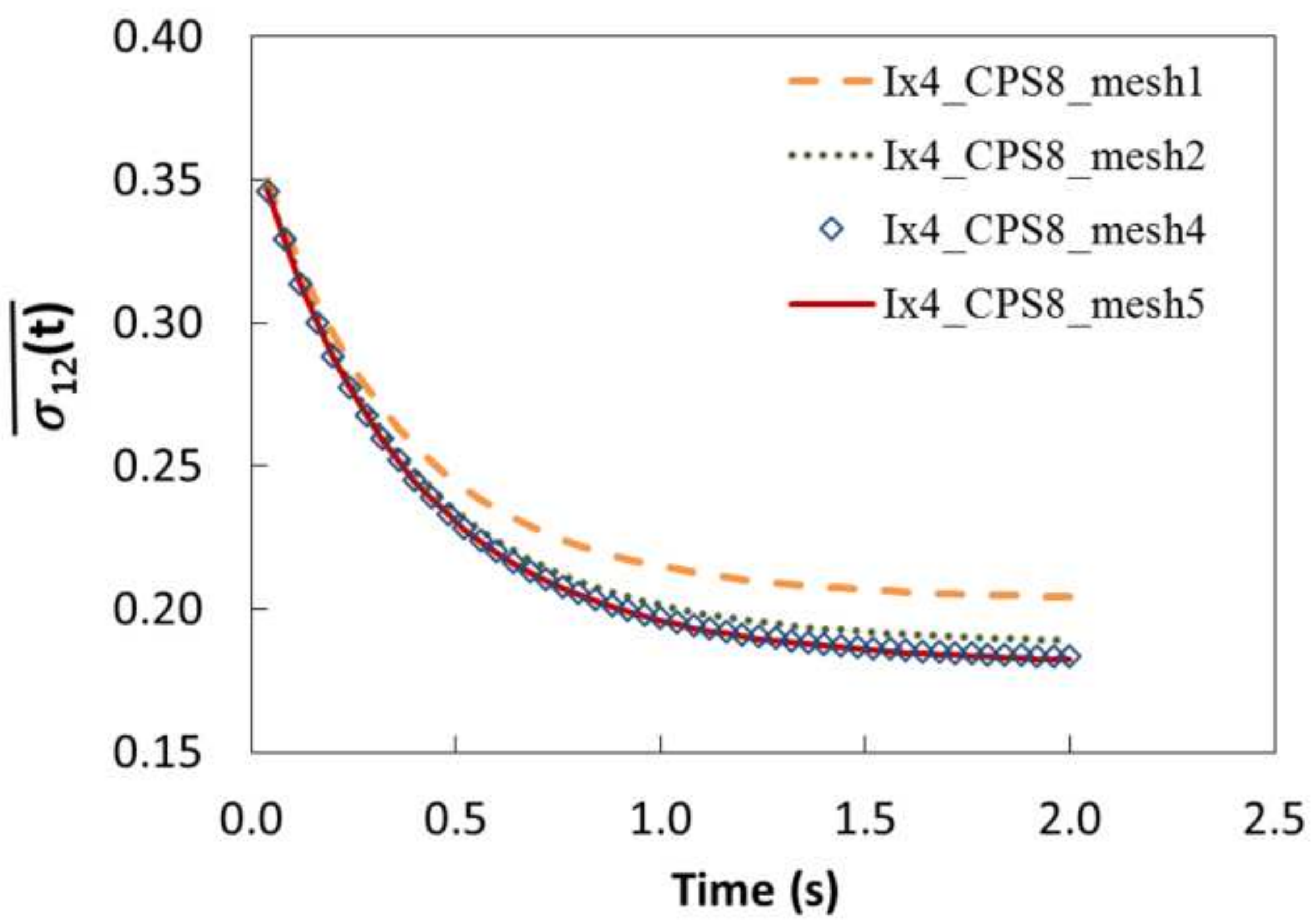


Click here to download high resolution image

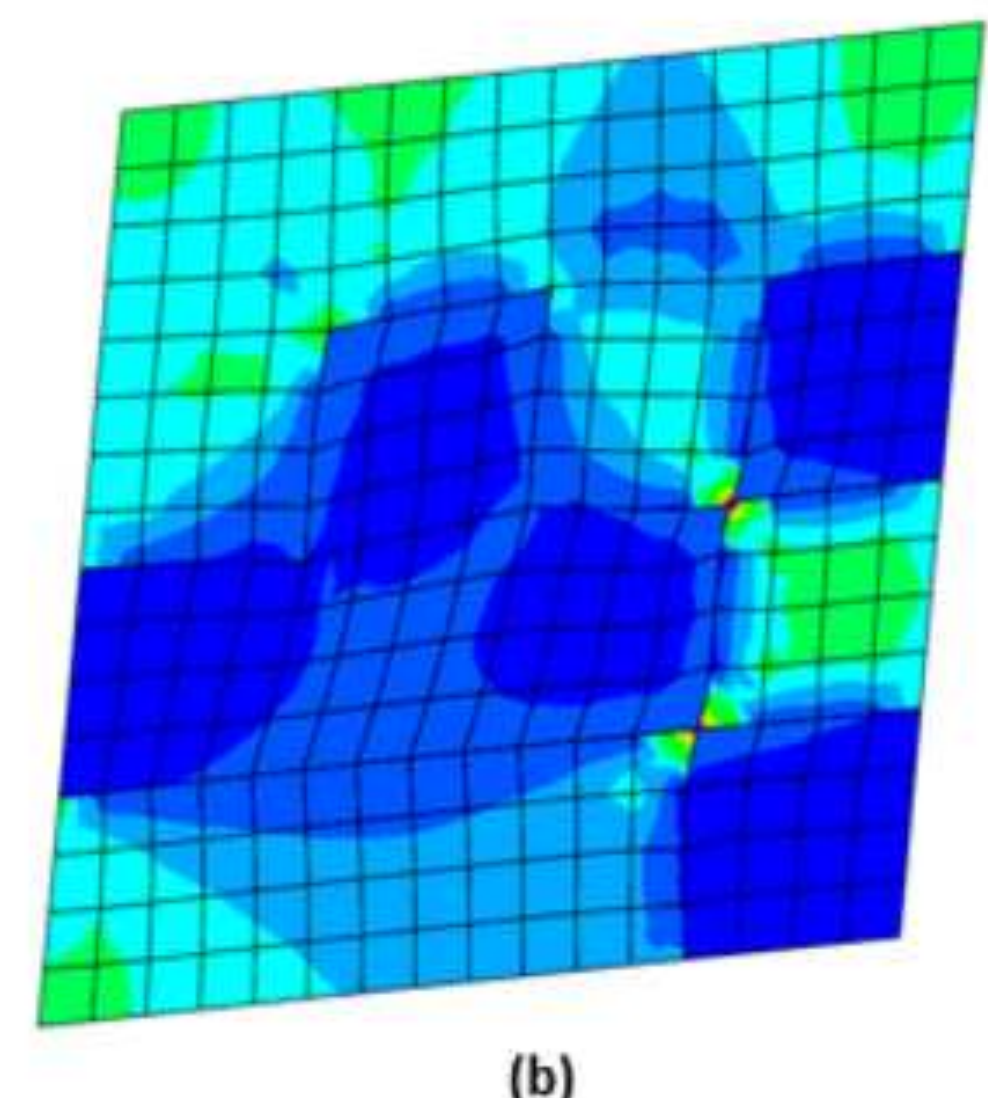

(b)

\begin{tabular}{|c|}
\hline $\begin{array}{l}\text { 5, } 512 \\
\text { (Awg: 754) }\end{array}$ \\
\hline 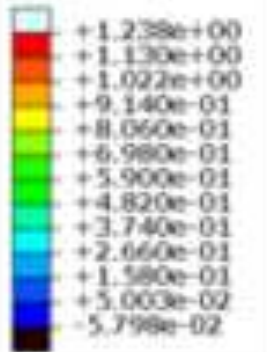 \\
\hline
\end{tabular}
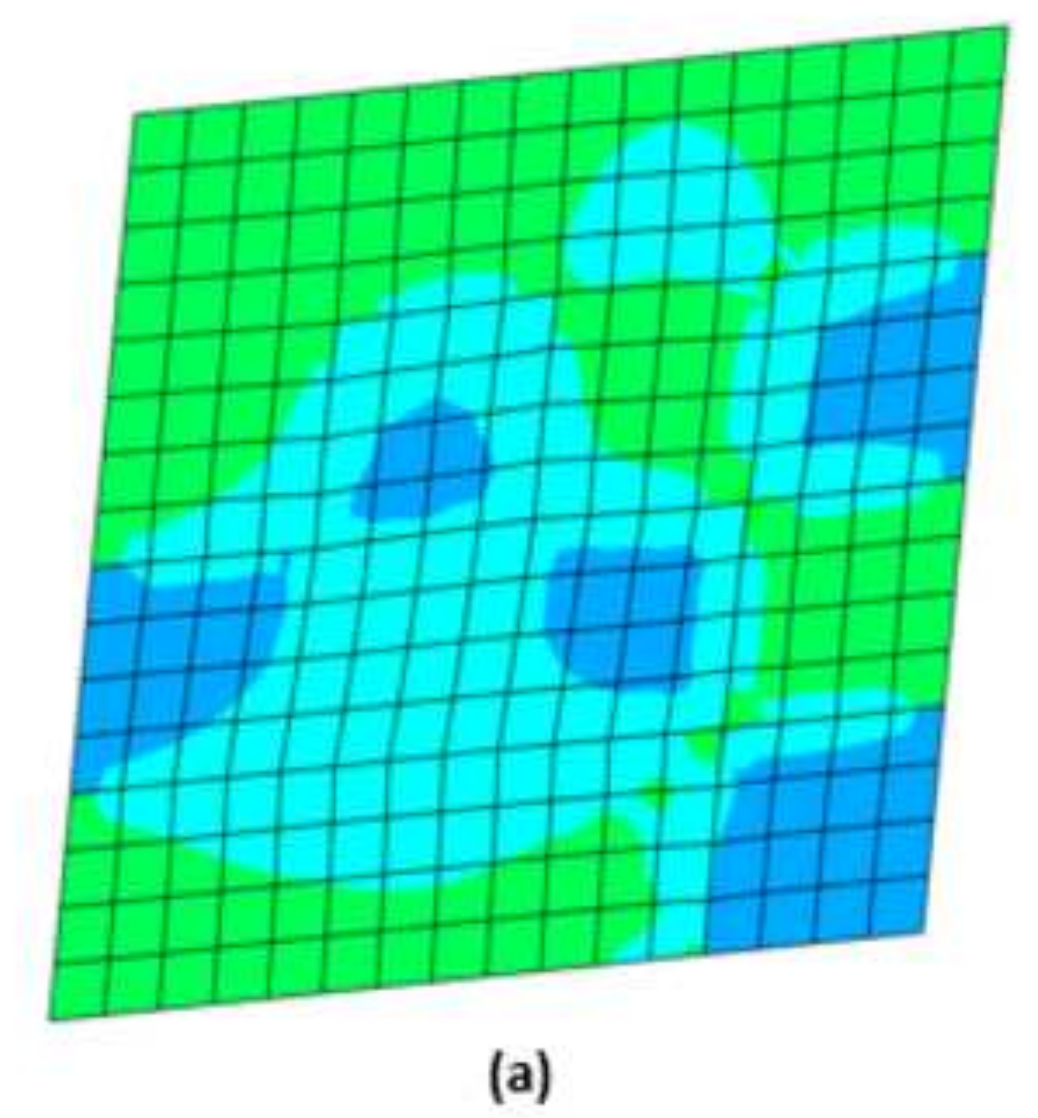

- nas (a)
(Awg: 7946 )

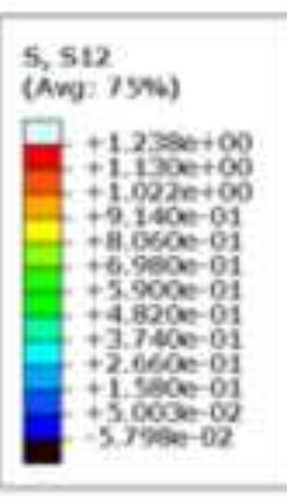

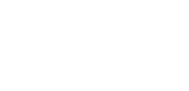


Click here to download high resolution image

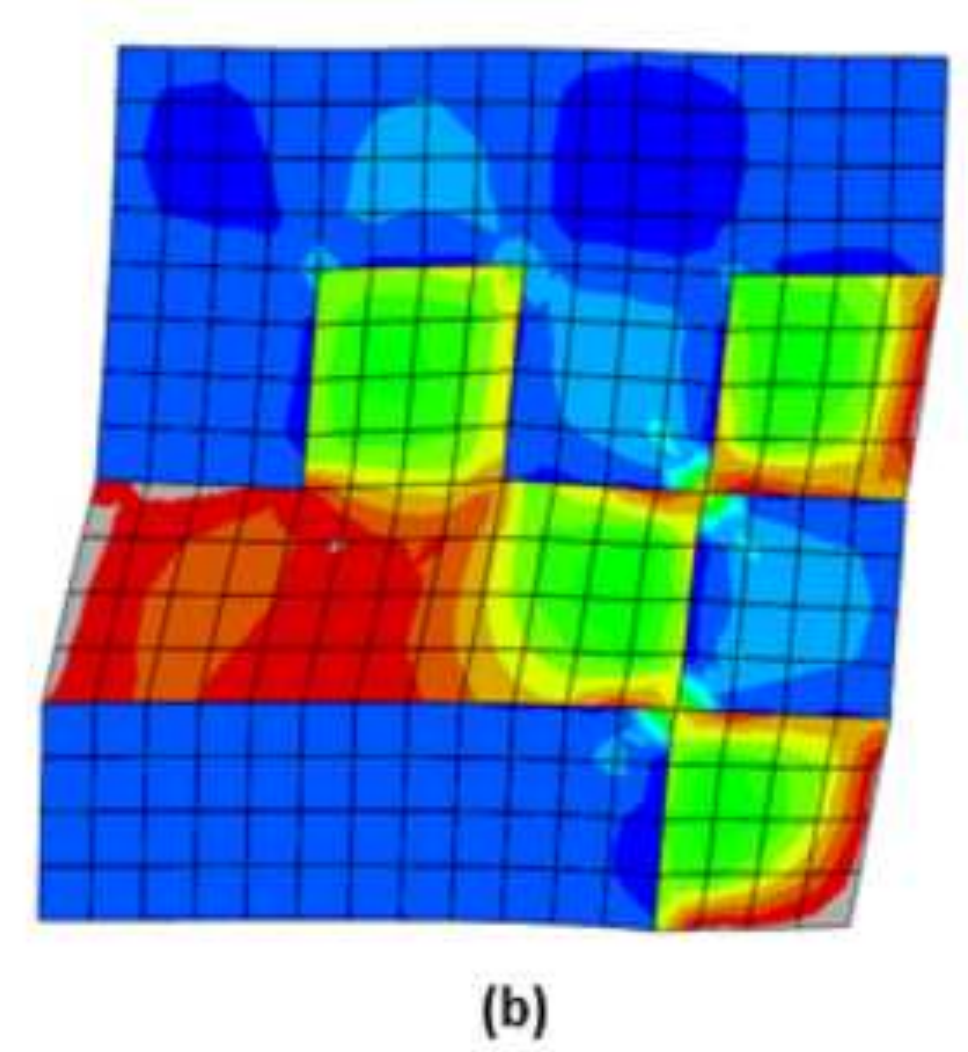

(b)

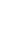

\section{(1)}

-

.

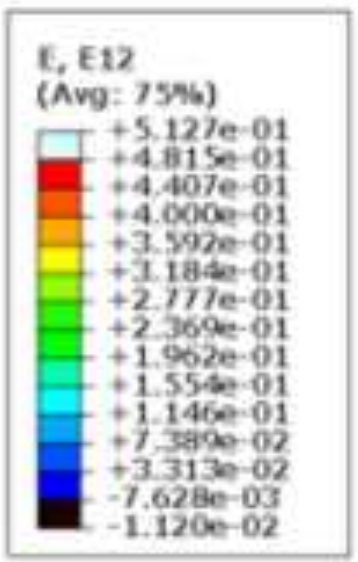

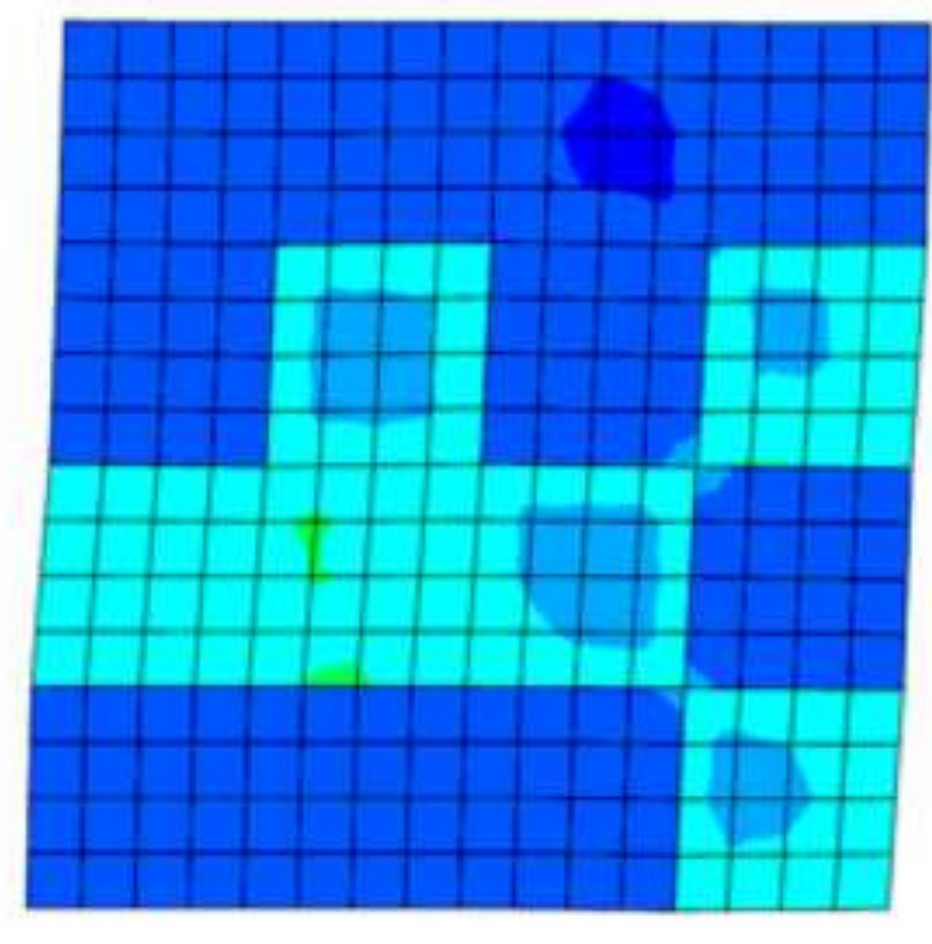

(a)

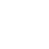


Click here to download high resolution image

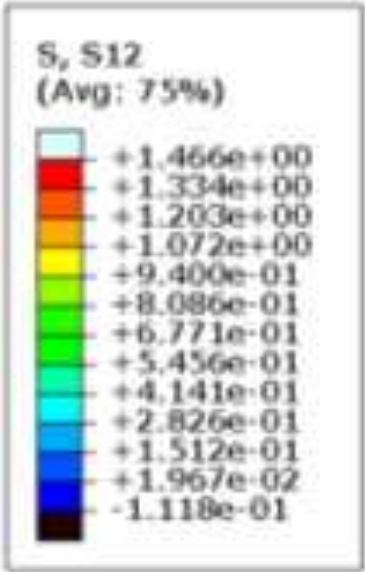

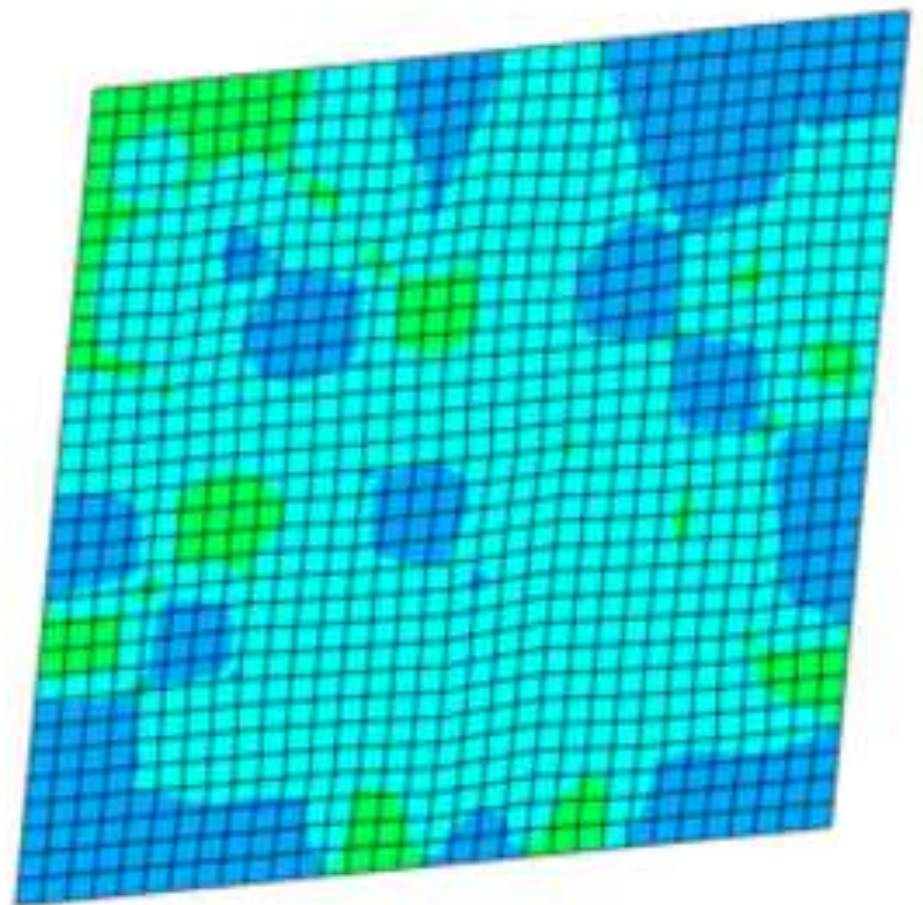

(a)

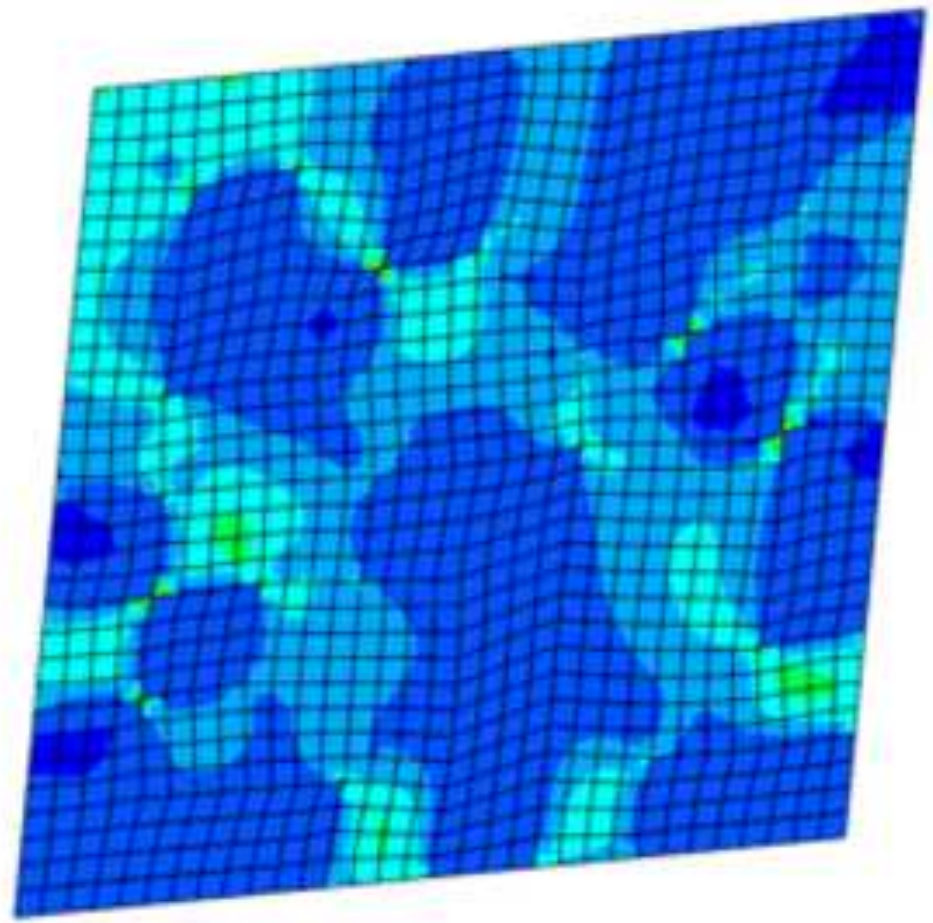

(b) 
Click here to download high resolution image

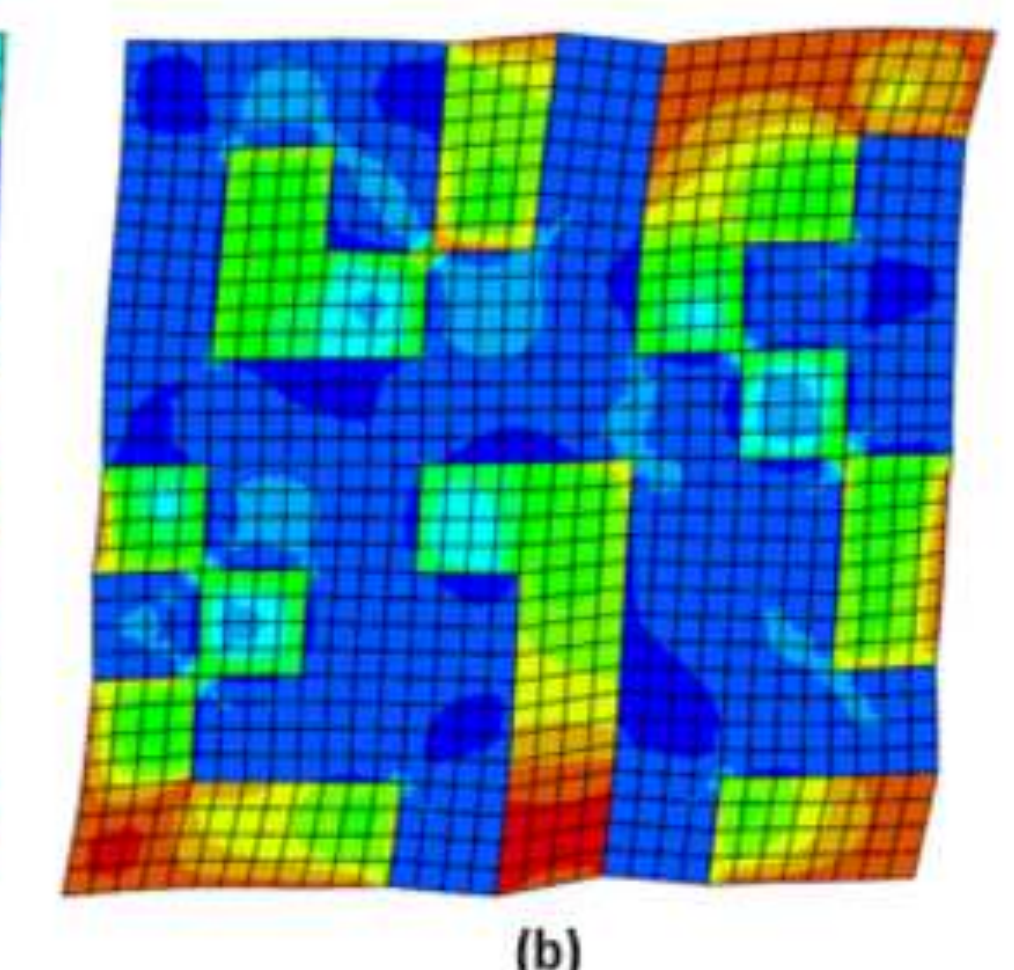

(b)

(n)

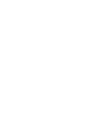

\begin{tabular}{|c|}
\hline $\begin{array}{l}\text { E, E12 } \\
\text { (Avg: 75\%) }\end{array}$ \\
\hline $\begin{array}{r}+5.802 \mathrm{e}-01 \\
+5.303 \mathrm{e}-01 \\
++4.805 \mathrm{e}-01 \\
+4.306 \mathrm{e}-01 \\
+3.807 \mathrm{e}-01 \\
+3.309 \mathrm{e}-01 \\
++2.810 \mathrm{e}-01 \\
+2.311 \mathrm{e}-01 \\
++1.813 \mathrm{e}-01 \\
+1.314 \mathrm{e}-01 \\
++8.15 \mathrm{e}-02 \\
+3.168 \mathrm{e}-02 \\
+1.819 \mathrm{e}-02\end{array}$ \\
\hline
\end{tabular}

(a)
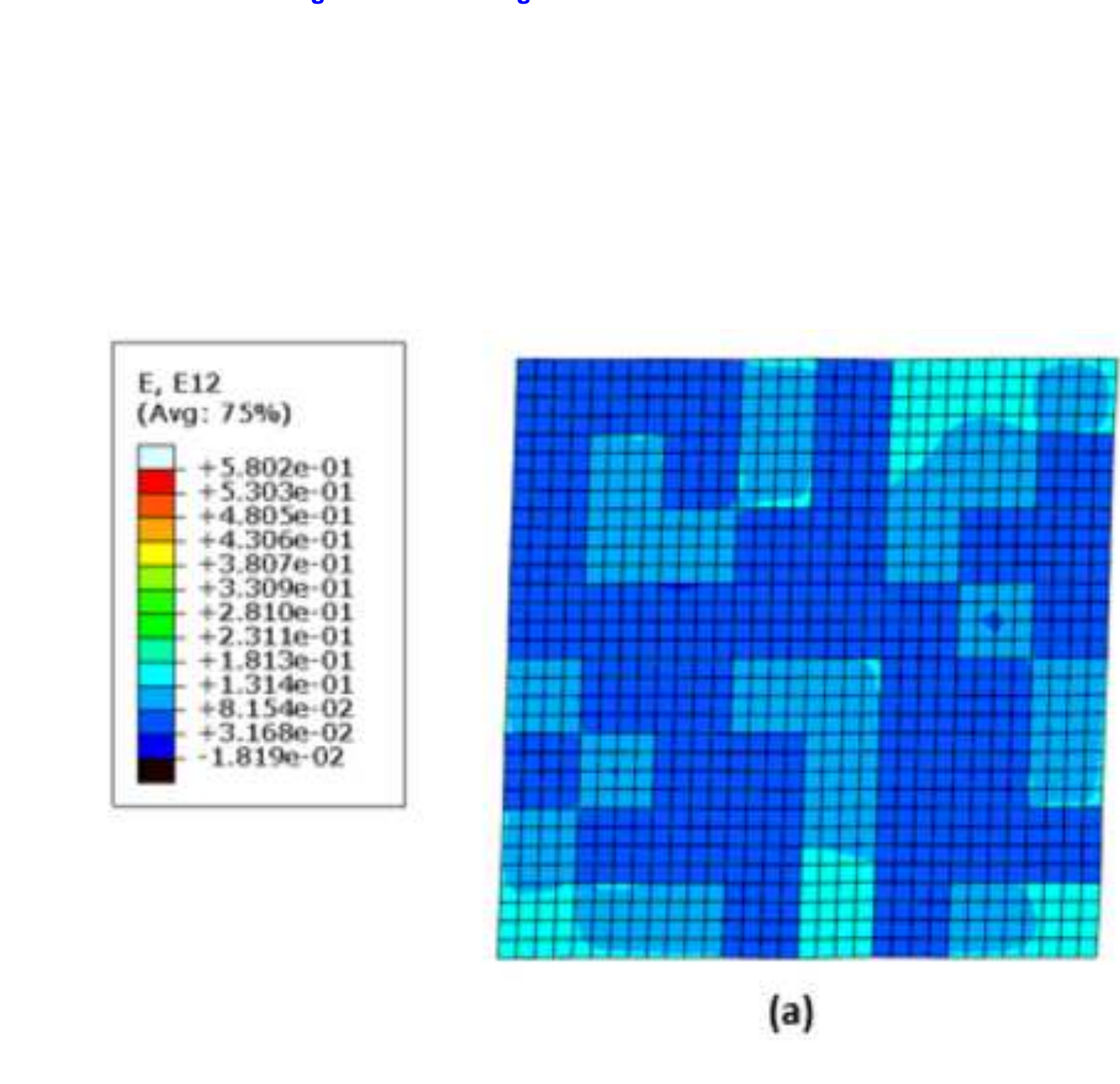

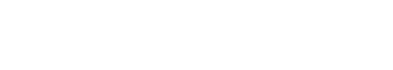


Click here to download high resolution image

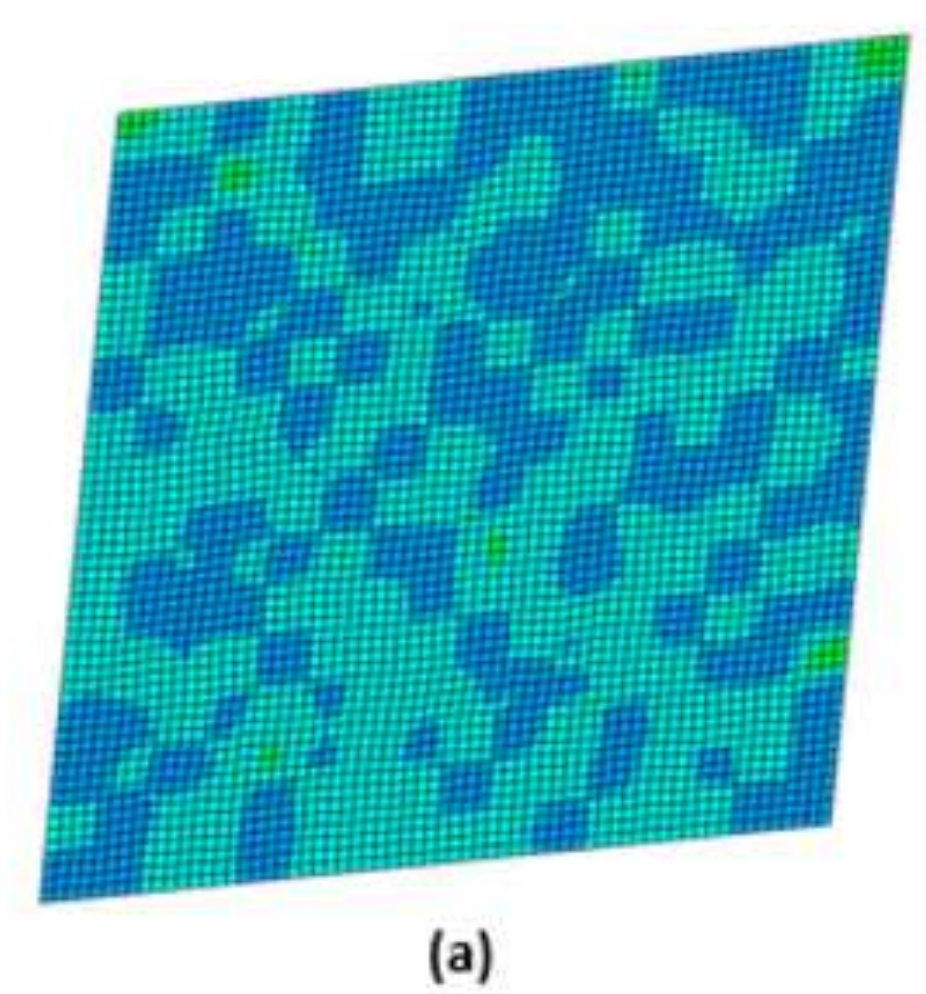

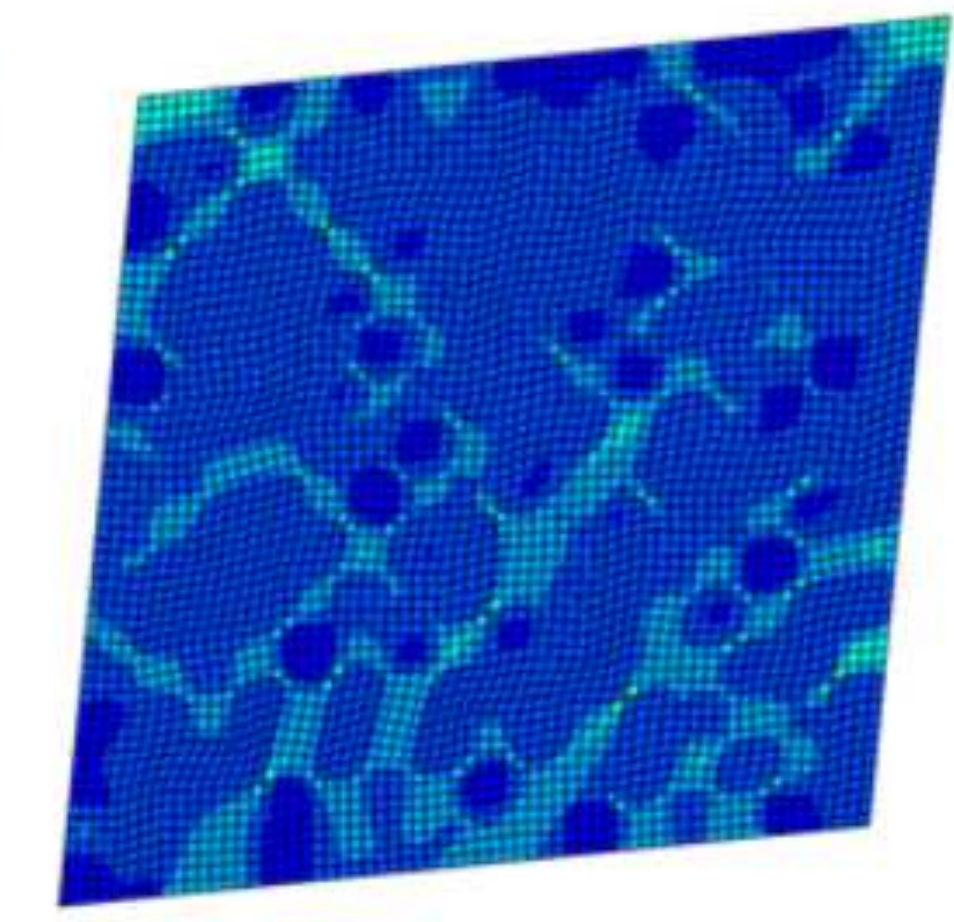

(b)

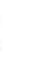

(a)

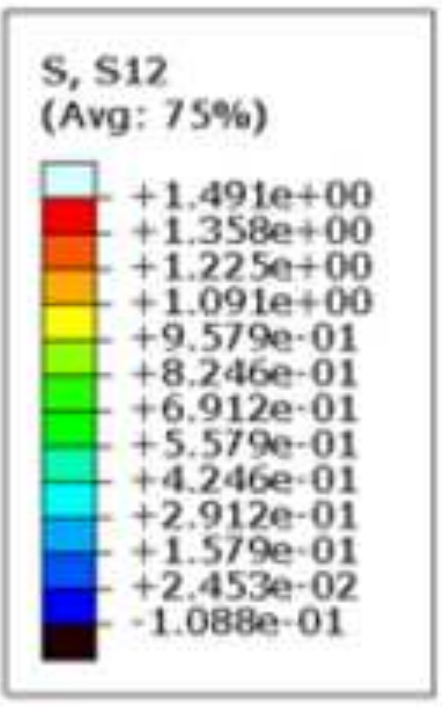


Click here to download high resolution image

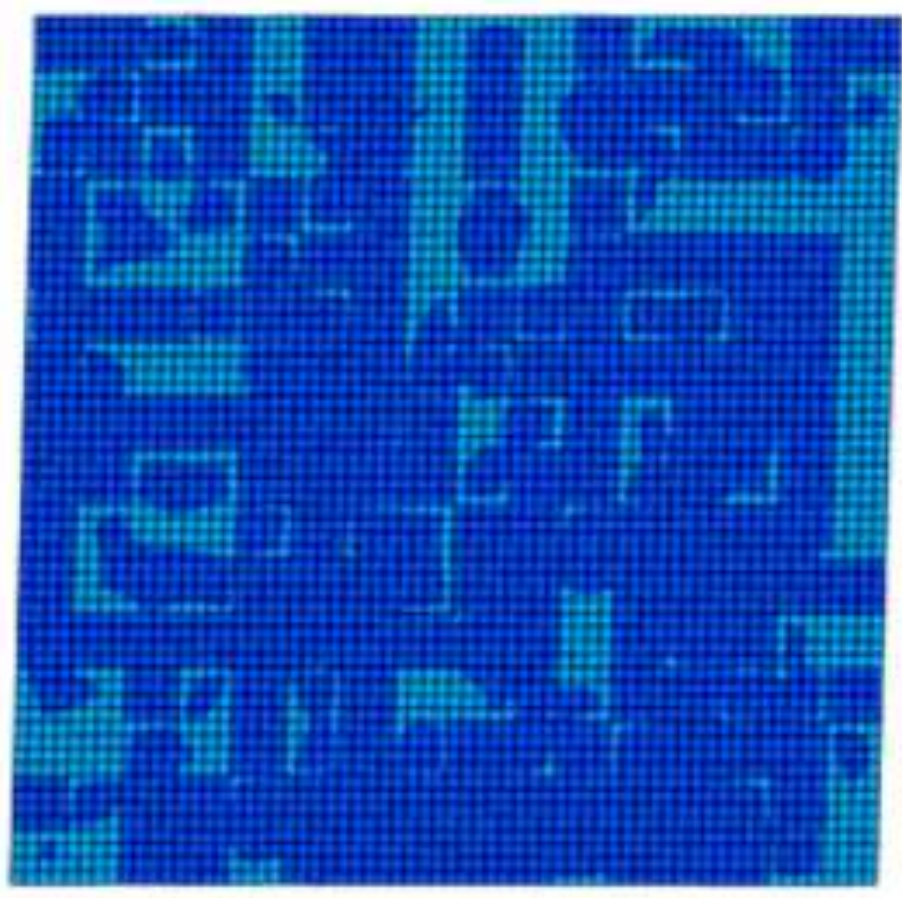

(a)

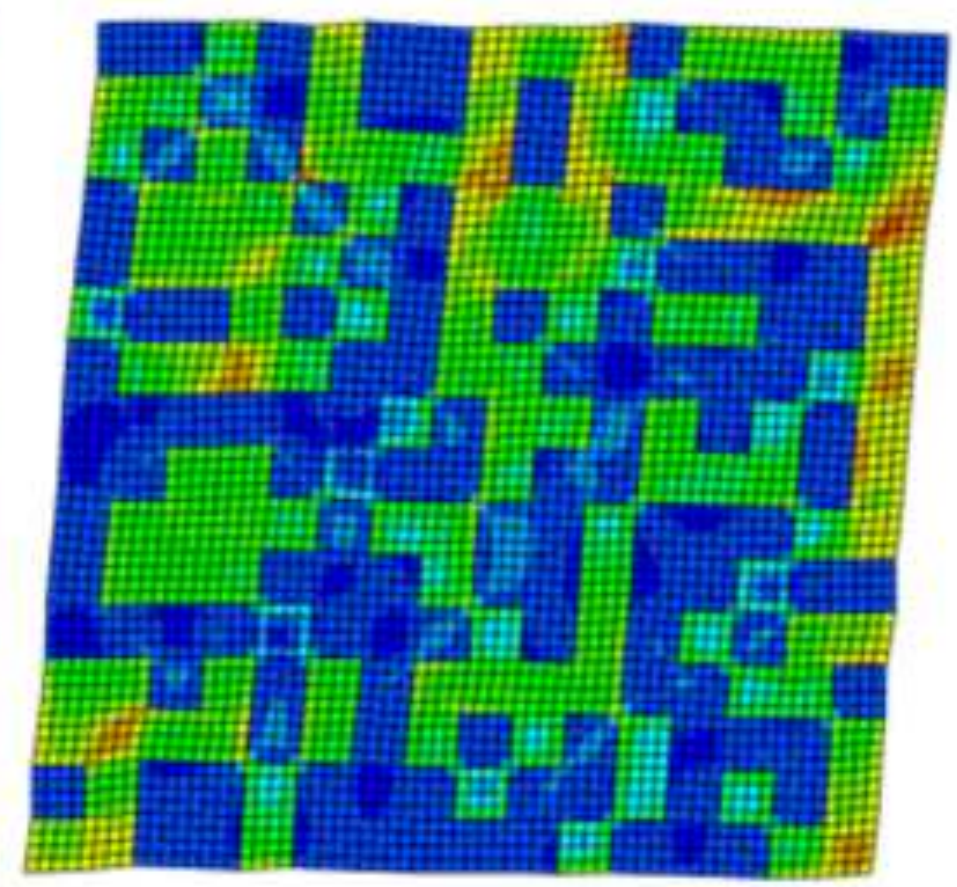

(b)

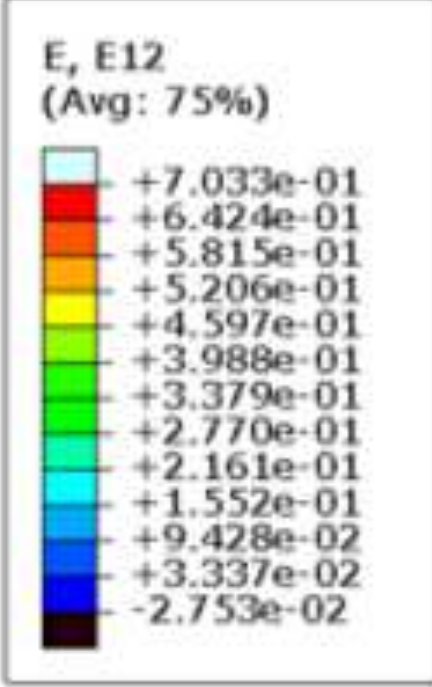

(Avg: 75\%)

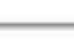




\section{Figure}

Click here to download high resolution image
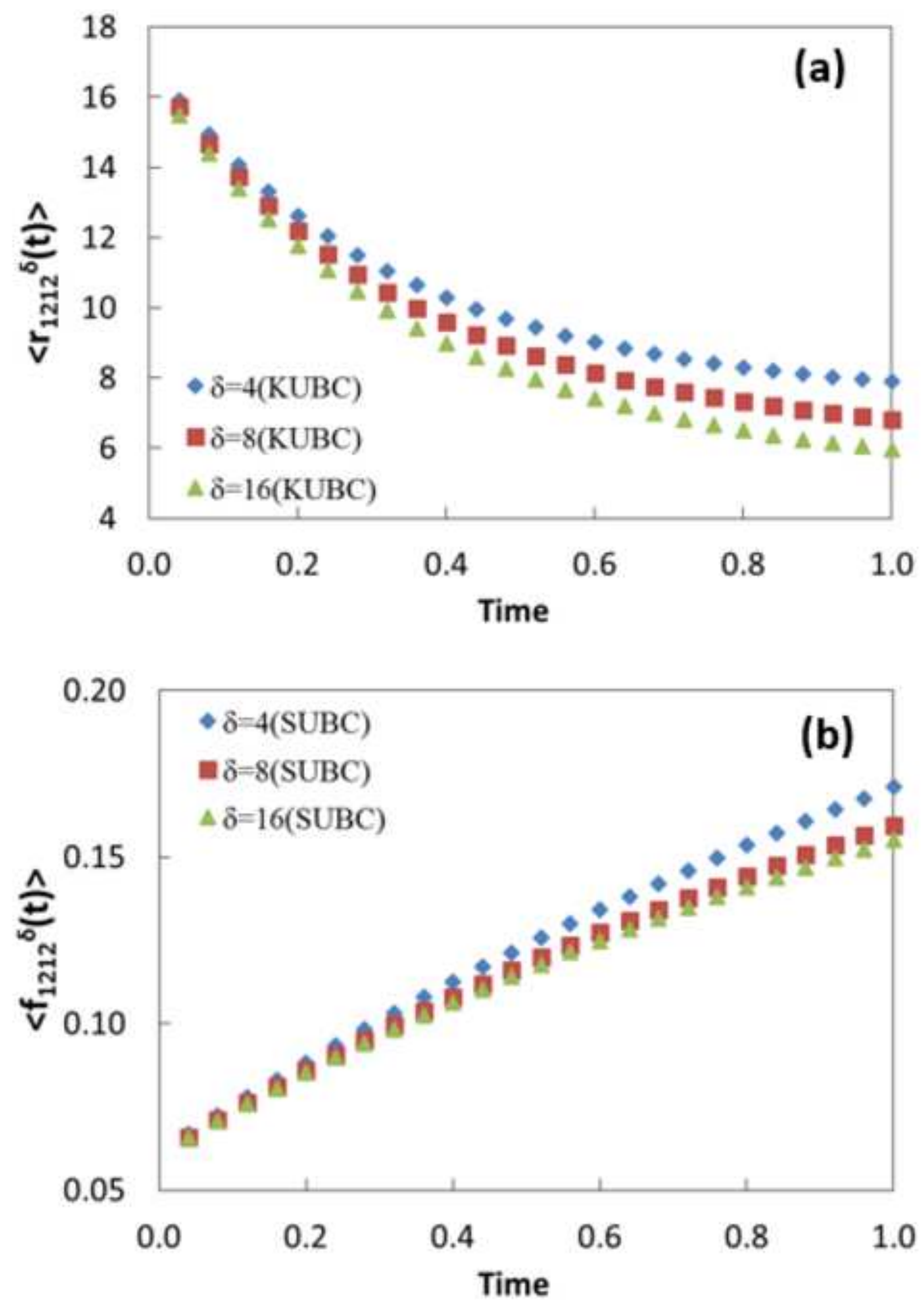


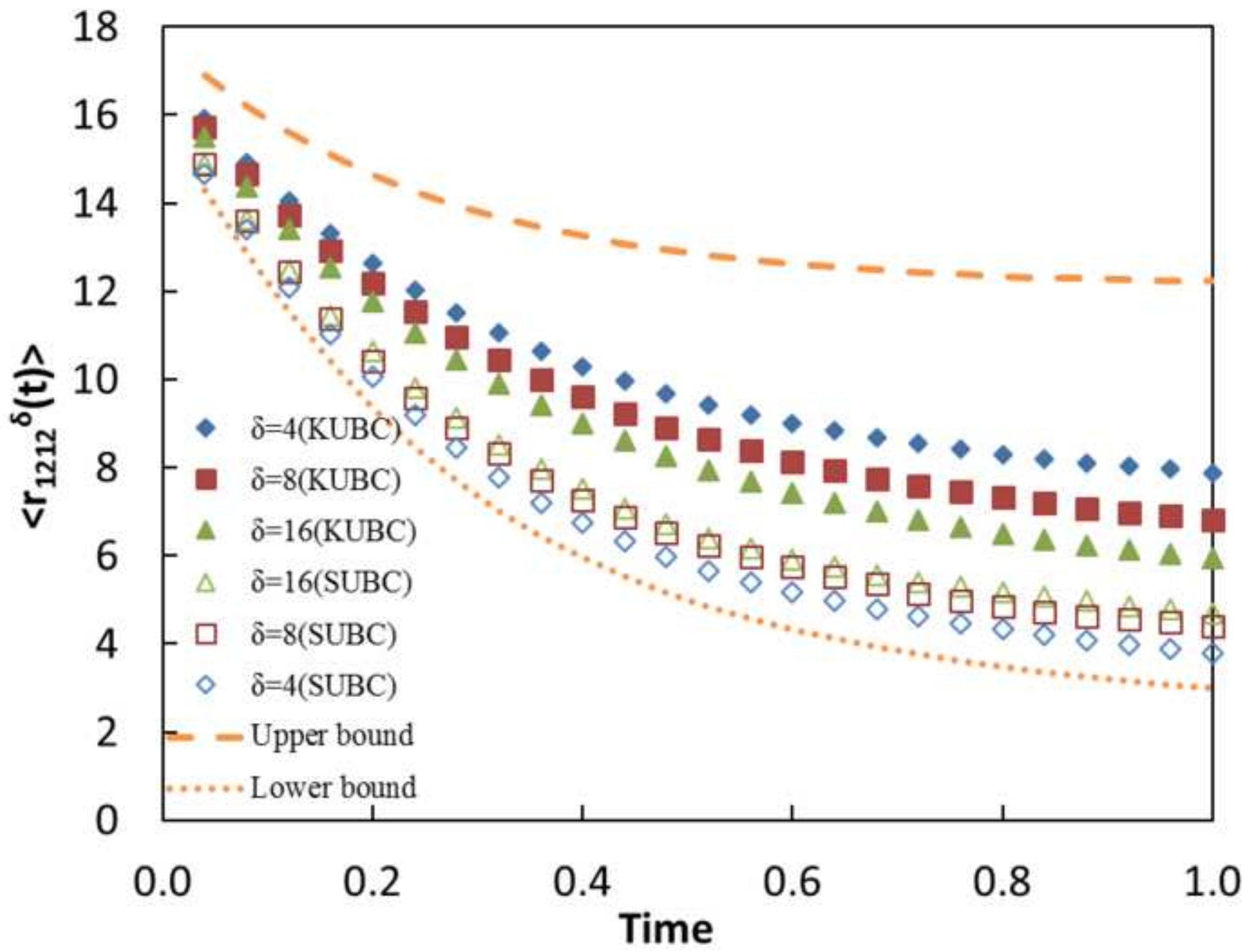




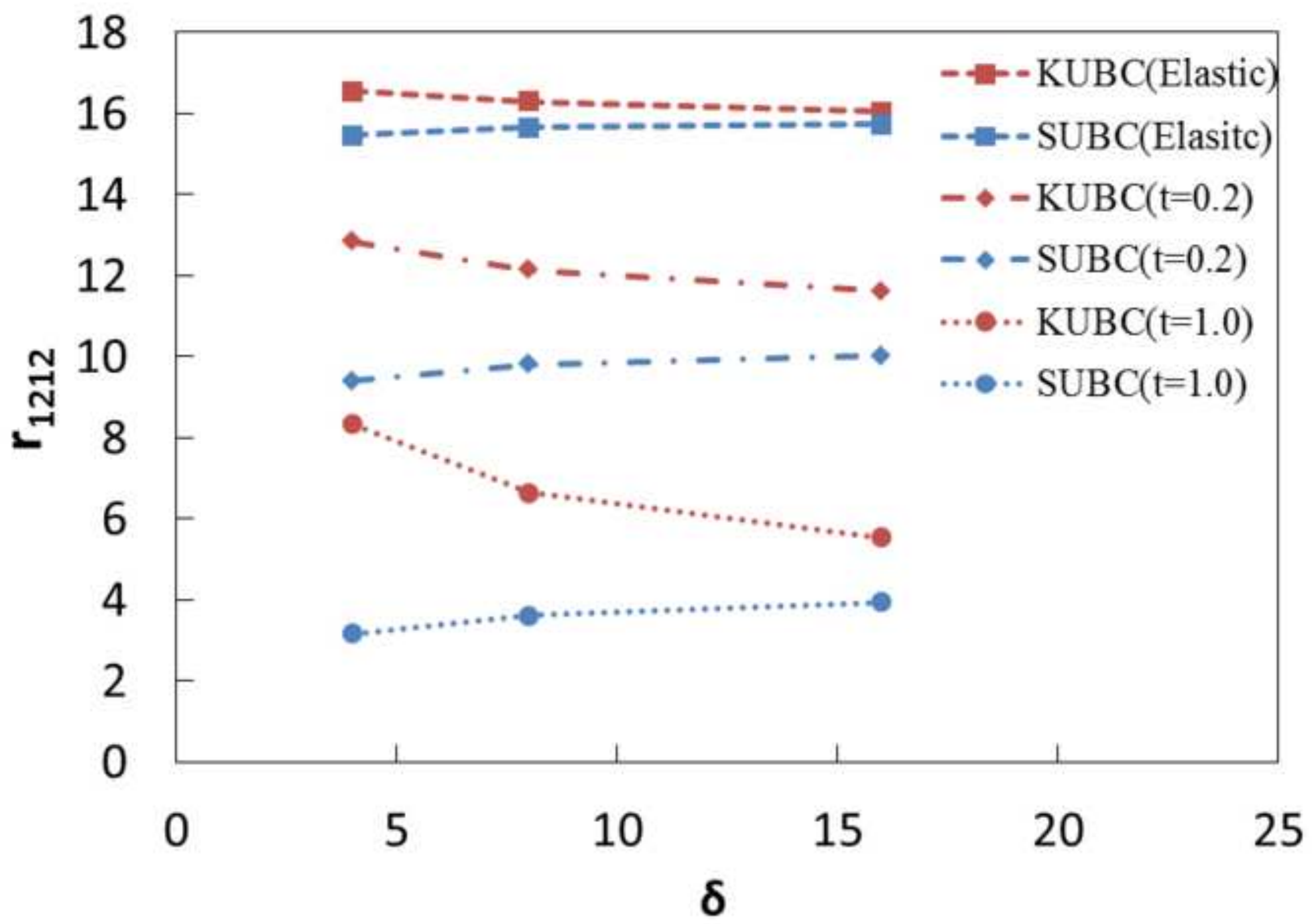

